\title{
Ueber Phenopyrylium
}

und hohere homologe und isologe Pyryliumringe; von Herman Decker und Theodor von Fellenberg.

(Eingelaufen am 19. Juli 1907.)

\section{Einleilung.}

\section{Theoretisches.}

Wenn heute eine Reihe von Verbindungen als Oxoniumverbindungen beschrieben werden, so bedarf dieser Standpunkt gewissermassen der Rechtfertigung. Seitdem nämlich die sauerstofffreien Salze des Triphenylcarbinols bekannt sind und als Carboniumverbindungen aufgefasst werden mussen, ist es $\mathrm{zweifel-}$ haft geworden, ob die früher beschriebenen Salze des Xanthyliums ${ }^{1}$ ), des Coeroxoniums ${ }^{2}$ ) und ibrer Schwefelisologen, die des Binaphtylbioxyliums und des Coerbioxyliums ${ }^{3}$ ), nicht vielleicht Carboniumverbindungen wären. Jenn sie enthalten alle die Atomconfiguration, die im Diphenyl- und Triphenylcarbinol vorkommt und alle ihre Umsetzungen wurden sich ebenso leicht unter der Zugrundelegung der Carboniumformel erklären lassen.

Zwei Umstände sind es gewesen, die uns veranlassten, dennoch an den Oxoniumformeln festzubalten.

1) B ü z ly und Decker, Ueber Xanthonium- und Thioxanthoniumverbindungen, Ber. d. deutsch. chem. Ges. 37, 2932.

2) Diese Annalen 348, 220; siehe auch Kapitel 4 dieser Abhandlung.

3) Decker, Ueber conjugirte Ringsysteme, Ber. d. deutsch. chem. Ges. 39, 3069 . 
Erstens ist es der grosse Unterschied, der in der Basicität zwischen den Triphenylcarbinolsalzen nnd den Derivaten des Phenylxanthenols besteht; währeud erstere schon in 70-procentiger Schwefelsäure nicht beständig sind und mit Salzsäure gar nicht entstehen, sind letztere noch in 10-procentiger Schwefelsăure beständig und lassen sich leicht mit Salzsäure darstellen. Ebenso sind die Coeroxonium- und Coerthioniumsalze mit verdünnter Salzsäure zu erhalten. Ueberall zeigen die Schwefelisologen merklich basischere Eigenschaften als die Sauerstoffkörper, in Uebereinstimmung mit der bekannten Thatsache, dass Thioniumsalze beständiger sind als Oxoniumsalze.

Zweitens ist es die Darstellung der Phenopyryliumsalze, bei denen die Carboniumformel eine sehr geringe Wahrscheinlichkeit besitzt, denn während die Oxoniumformel (I)

I.



P'henopyryliumchlorid
II.

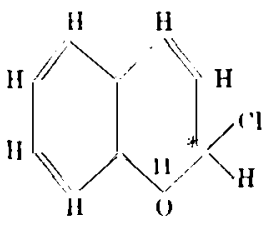

Phenopyranolchlorid

die Eigenschaften dieser Salze, ihre grössere Basicität und ibre Farbigkeit als durchaus verständlich erscheinen lässt, und auch warum sie die grösste Aehnlichkeit mit den Salzen des quaternären isologen Chinoliniums aufweisen, hat die Carboniumformel dieser Verbindungen das Unbequeme, dass sie uns zwingt, 1ur das in der Furmel mit einem Sternchen versehene Koblenstoffatom basische Kigenschaften anzunehmen und zwar sehr viel stärker basische als für den Methankublenstoff des Triphenylcarbinols. Zwischen der Structur der Triphenylcarbinolsalze und eines derartigen Körpers von der Formel II kann man nichts Gemeinsames entdecken, was den Schluss auf das analoge basische Verhalten des Kohlenstoffatoms des letzteren recht- 
fertigen würde. Mit anderen Worten: würde nicht durch vorliegende Arbeit die Salznatur der bier Phenopyrylium genannten Verbindungen bekannt geworden sein, so würde wohl Niemand vermuthen künnen, dass ein Körper von der Formel II ein Salz vorstellt und gelb gefürbt ist. Nur rom Standpunkte der Oxoniumtheorie ist dieses wach der Formel I selbstverständlich und vorauszusehen; wirklich sind auch die beiden in dieser Arbeit ausgeführten Synthesen der Phenopyryliumsalze unter Anwendung der Oxoniumtheorie entworfen worden.

Ist nun aber vierwerthiger Sauerstoff für die Phenopyryliumsalze angenommen, so muss die Analogie der Eigenschaften dieser Salze mit denen des Nanthyliums, Thioxanthyliums, Coeroxoniums, Coerthioniums u.s. w. diese als höhere Glieder derselben Reihe von Ringen kennzeichnen. Wie die Phenopyryliumsalze als Isologe der Chinoliniumsalze, erscheinen also jene Reihen als Isologe der Acridinium- und Coeramidoniumsalze, eine Auffassung, die ihre vollkommene Bestätigung in dem Verhältnisse der Farbigkeit und der Basicitäten dieser drei isologen Keihen findet.

Allerdings wäre es möglich, von der unverkennbaren $\mathrm{Zu}$ sammengehürigkeit der Stickstoff-, Schwefel- und Sauerstoffisologen der drei Reihen ausgehend, umgekehrt in den Coeramiloniumverbindungen und in den Acridiniumverbindungen den Säurerest an das betreffende Kohlenstoffatom zu schreiben und sie alle als Carboniumverbindungen aufzufassen. Diese Annahme hat den Vortheil, einerseits den drei isologen Reihen und andererseits den Triphenylcarbinolsalzen, d. b. den wabren Carboniumverbindungen, die gleiche Structur zuzusprechen, aber sie zwingt natürlich auch zur Auffassung der Chinolinium- und Pyrydiniumverbindungen als Carboniumverbindungen. Wir würden nach dieser Auffassung ein abgeschlossenes System besitzen, das zugleich mit der Verneinung des vierwerthigen Saverstoffs und Schwefels auch auf den Gebrauch des fünfwertbigen Stickstofis in den cyklischen Verbindungen uberbaupt verzichten würde, das sich auch in Berug auf die clemischen Reactionen 
der Salze mit Erfolg vertheidigen ${ }^{4}$ ) lassen und die Analogie, die in den Eigenschaften, Habitus und Verbalten aller Oniumverbindungen besteht, in verwandten Formelbildern berworheben würde.

Doch wird durch eine weitere unvermeidliche Schlussfolgerung eine derartige Auffassung ad absurdum gefüht, wenigstens so lange wir die Fesseln der Structurtheorie nicbt abstreifen wollen. Zwischen den quartăren Salzen des Pyridins und den quartären Salzen der aliphatischen Basen, z. B. Tetrametbylammonium, besteht in Bezug auf ihre Eigenschaften und chemisches Verhalten eine derart weitgebende Achnlichkeit, dass wir sie unmöglich zu zwei verschiedenen Körperklassen rechnen könuen. Wenden wir aber die abgeleitete Carboniumformel für die quaternären Salze des Pyridins auch auf das 'T' tramethylammoniumjodid an, und schreiben den Säurerest zum Koblenstoff, so zerfällt unser Formelbild in zwei Moleküle. I)as wurde mit Worten heissen: „Die quaternären Ammoniumsalze sind molekulare Verbindungen", wir kommen also auf deu verlassenen Standpunkt zurück. Man könnte hier auch leicht, wenn man sich durch die Grenzen der Structurtheorie nicht binden lässt, Folgerungen ziehen, die dem Anschaungekreise Werner's nahe sind, und die sich auf die Natur der Ammoniumbindungen überhaupt beziehen. Dieser Weg ist auch vielleicht der richtige, um die Analogie, die zwischen all den Oniumverbindungen thatsăchlich besteht, durch Formeln auszudracken. Er fuhrt uns aber uber das Gebiet der klassischen Structurtheorie hinaus und findet in ihrer Formelsprache keine Ausdrucks. möglichkeit ${ }^{5}$ ).

4) Nicht aber was die Ursache der Basicität eines Kohlenstoffatoms anbetrifft, die in der Nähe und Anhäufung negativer Phenylreste gesucht wurde; vergl, die Carboniumformeln der Pyridiu- und Chinolinsalze (siehe Formel II obeu).

5) Die Unaulinglichkeit der Formelsprache hat sowohl Adulf von Baeyer als anch Werner zur Einführung von neuen Zeichen geführt, welche eine besondere art von Hindungen ansdricken 
So lange wir also in früberer Weise das Totramethylammoniumjodid mit fünfwerthigem Stickstoff durch das alte Formelbild zum Ausdrucke bringen, mussen wir auch die. Ammonium-, Oxonium- und Thioniumformeln anerkennen, wie sie bisher gebraucht worden sind, und wie ich sie auch hier anwende.

\section{Ueber Oxoniumsynthesen.}

Die untersuchten Reihen enthalten alle dic sauerstoff- oder schwefelhaltigen Sechsringe des Pyryliums ${ }^{6}$ ) (I) und Thiopyryliums (III). Diese Ringe sind dem Benzolringe analog gebaut und dem Pyridin in seinen Salzen isolog.

sollen. Verfolgt man den oben angedeuteten Gedankenweg weiter, so kommt man zu einer Auffassung, von der aus gesehen Ba eyer's und Worner's Formeln das Gleiche suchend, erscheinen. Die unverkenubare Analogie der Triphenylcarbinolsalze und der Phenylxanthyliumsalze, die mit unseren gewohnlichen Structurformeln nicht zum Ausdrucke gebracht werden kunn, wïde vielleicht hier in folgendem Bilde ilıre Erklärung finden:
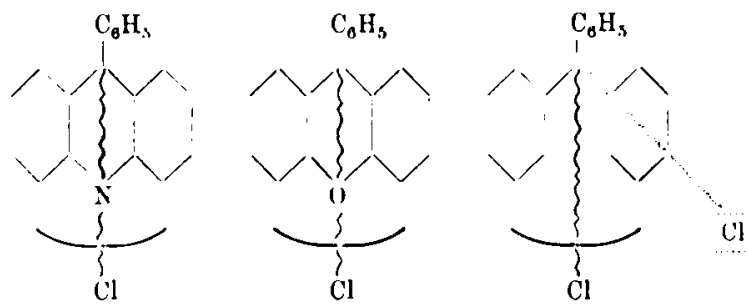

Man muss sich das ionisirbare Halogenatom an der Zickzackbindung ähnlich wie ein Gewicht an einer Spiralfeder hängend denken, es ragt in die zweite Sphäre (wie durch den Kreisbogen in der Formel angedeutet ist) hinaus, befindet sich aber bei den Xanthylium- und Acridiniunverbindungen auch zugleich in der Allziehungssphäre des Sauerstoff- und Stickstoffatoms. Bewegt man dieses Atom um seinen Hängepunkt um $60^{\circ}$, so gelangt man zur Formel von Kehrmann für die Triplenylcarbinolsalze, d. h. das Chlor gelungt danu in die Sphäre des p-Kohlenstoffs.

c) Vergl. die Tabelle. 


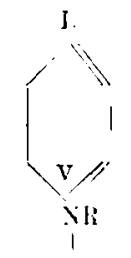

Pyridinium

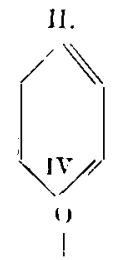

ryylium

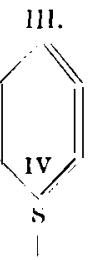

Thiopurylium.

Verbindungen, die den einfachen Pyrylium- oder Thiopyryliumring enthalten, haben wir vorläufig noch nicht darstellen können, dagegen sind die höheren complexen Ringe vertreten ${ }^{7}$ ). Gleich dem Benzol- und Pyridinring zeichnet sich der Pyryliumring uberall durch seine grosse Beständigkeit (aber nur in sauren Lösungen) und durch die Tendenz, sich durch Ringschliessung zu bilden, aus.

Die Oxoniumtheorie gestattet, eine Reihe von Synthesen theoretisch zu construiren, von der Voraussetzung ausgehend, dass in Gegenwart von Mineralsäuren, also z. B. in Gegenwart von concentrirter Schwefelsäure, ein Sauerstoffatom wie ein vierwerthiges basisches Element functionirt, etwa wie die secundäre Aminogruppe, und entsprechende Condensationew unter Ringschliessungen eingeht. Die entstehenden Oxoniumsalze besitzen auch bei höherer Temperatur eine genügende Beständigkeit, um als Endproduct der Reaction aufzutreten.

In den folgenden Kapiteln sind Beispiele zu derartigen Synthesen ${ }^{8}$ ) zusammengestellt, die wir von diesem Gesichtspunkte unternommen und auch ausgeführt haben.

Im ersten Kapitel ist die Synthese von Phenopyryliumderivaten, den Sauerstoffisologen der Chinoliniumverbindungen,

7) Vergl. die Tabelle.

3) Das hier mitgetheilte experimentelle Material war bereits im Sommer 1906 abgeschlossen. Obgleich es in dieser Gestalt noch manche Liicke und Ungleichmässigkeit in der Behandlung zeigt, glaub ich doch, dass es in seiner liesammtheit einen genügeuden Leberblick iiber das (jebiet der stickstoffreien Uxoniumbasen gestattet, um seine J'ublication nicht noch weiter hinausschieben zil sollen.

H. 1 . 
durch Condensation von Salicylaldehyd mit Aldehyden und Fetonen und nachfolgender Ringschliessung, beschrieben. Diese Darstellungsweisen verlaufen glatt nach dem Schema
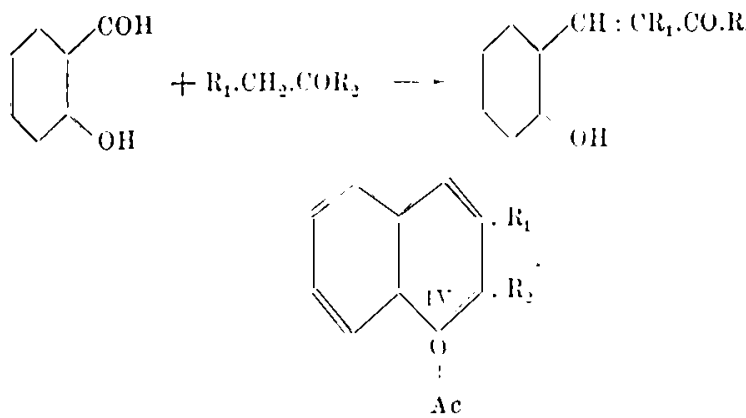

Zu den gleichen Verbindungen gelangt man durch die Einwirkung von Alkyl- oder Arylmagnesiumhalogeniden auf Cumarin:

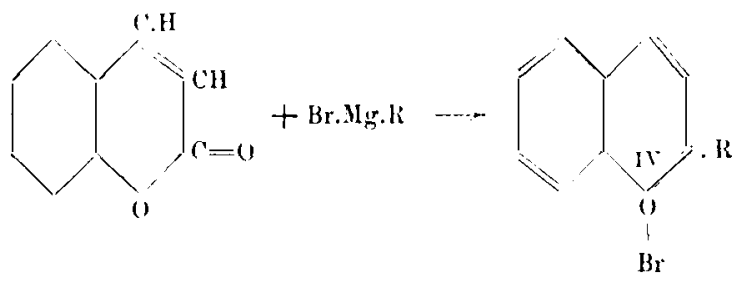

Die beiden Synthesen sind hier nur an einigen Beispielea erläutert. Es lasseu sich aber durch sie eine grosse Anzahl ron Derivaten, Homologen und Substitutionsproducten des neuen Kinges herstellen. Besonders die erste Synthese macht die Phenopyryliumverbindungen zu leicht zugänglichen Körpern und ist in mancher Hinsicht des Ausbaues fäbig. Dieses Kapitel ist also die vorläutige Bearbeitung eines Gebietes, das noch eine reiche Ernte verspricht. Insbesondere soll die Darstellung der entsprechenden Schwefelisologen, die noch unbekannt sind, versucht werdeu.

Im zweiten Kapitel sind mehrere neue Beispiele für die von Decker und $B u ̈$ u $z y^{9}$ ) früher aufgefundene Darstellung von mesosubstituirten Xanthyliumdericaten angefuhrt:

9) Ber. d. deutsch. chen. Ges. 37, 2932 (1904). 


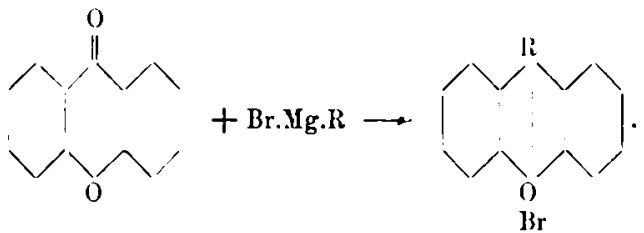

Daran anschliessend sind rom Fluoran sich ableitende Salze der Xanthyliumcarbonsäure beschrieben und die Ursache ihrer auffallend geringen Basicität, welche eine Ausnahme in der Reihe der Oxoniumbasen zu bilden schien, zu erklären versucht.

Das dritte Kapitel bringt Versuche in der Coeroxoniumund Coerthioniumreike unter Anwendung der fruber mit Ferrario ${ }^{10}$ ) gefundenen Synthese aus Fluoransalzen zur Darstellung von Dimethylcoeroxoniumsalzen:

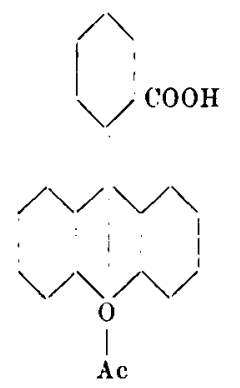

Salz des Fluorans

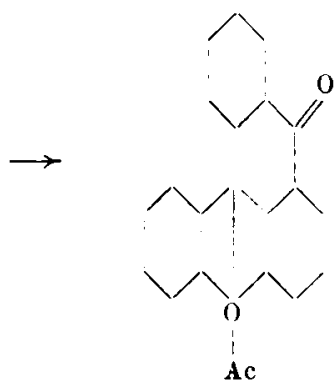

Salz des Coeroxoniums.

Eine andere früher ${ }^{30}$ ) gemeinscbaftlich mit Ferrario, Laube und Wursch ausgearbeitete Synthese ist ebenfalls zur Darstellung von homologen Coeroxonium- und Coerthioniumsalzen benutzt worden:

10) Diese Annalen 248, 210: l) ecker, Ueber Coeroten. 
und höhere homologe und isologe Pyryliumringe. $\quad 289$

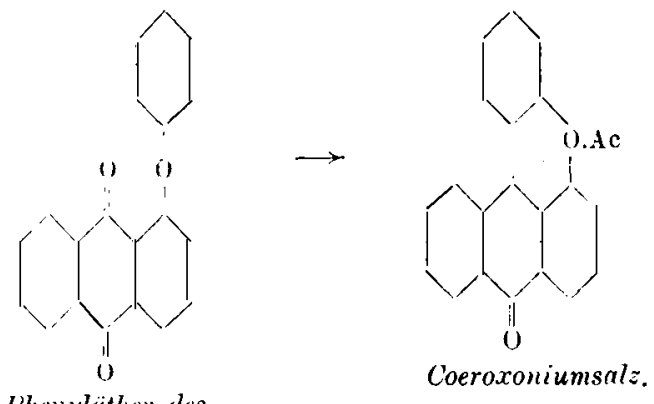

I'henyläthor des

Erythrooxyanthrachinons

Ausserdem sind in diesem Kapitel einige Derivate des einfachen Coeroxoniums beschrieben ${ }^{10}$ ).

Das vierte Kapitel ist der weitere Ausbau der Anthrachinonaryläther- und Thioäthersynthese, welche hier zur Darstellung vou hochmolekularen complexen Dioxonium- und Dithioniumverbindungen benutzt worden ist. Ein Beispiel sei durch folgende Formel gegeben:
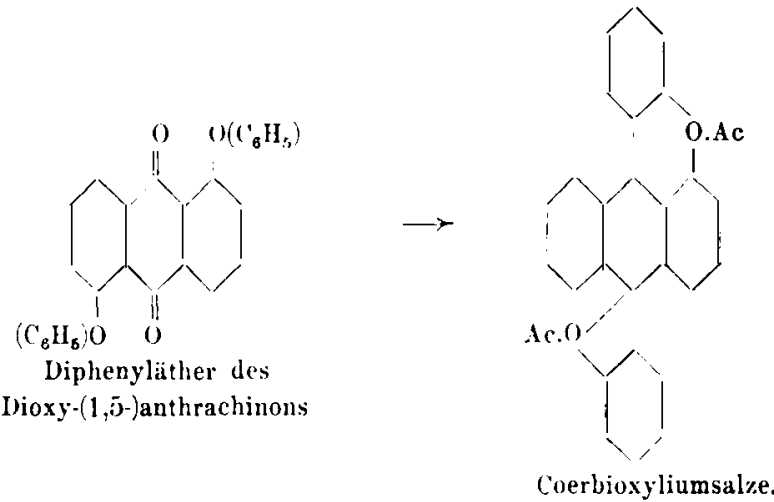

Diese letzten interessanten Reihen sind leider nicht sehr zugäıglich und ist deshalb die detaillirte Ausarbeitung nicht nach Wunsch möglich gewesen. Wir haben schliesslich auch vorläufig davon abgesehev, weil die Frage nach den springenden Bindungen in conjugirten Ringsystemen ${ }^{11}$ ), welche aus den

11) Ber. d. deutscl. chem. (ixs. 39, 3069. 
Oxydations- und Reductionserscheinungen dieser Salze abgeleitet wurde, unterdessen an dem 2,f-Naphtochinon von Willstätter und an den verschiedenen complexen Anthrachinonküpenfarbstoffen ron Roland $\mathrm{Sch}$ oll eine genügende Bestätigung und ein dankbares Material gefunden hat.

Die skizzirten Oxoniumynthesen verlaufen, sobald die richtigen Bedingungen gefunden und eingehalten werden, ausserordentlich glatt. Es ist auch anzunehmen, dass sich den beschriebenen neue Oxoniumsynthesen bald anschliessen werdeu. Eine Anzahl von Farbenreactionen, die Aldehyde, Ketone und Phenole mit Schwefelsäure geven und die seit langer Zeit bereits bekannt und beschrieben sind, werden ebenfalls auf derartige Oxoniumsynthesen unter Bildung von Pyryliumringen zurückzuführen sein. Verschwindet die Farbe dieser Lösungen in concentrirter Säure bei Verdünuung mit den gleichen bis doppelten Gewichte Wasser nicht, verblasst sie aber schnell oder allmühlich bei einer Verdünnung mit dem 50-fachen bis 100-fachen Gewicht, so ist das Vorhandensein eines Oxoniumsalzes wahrscheinlich.

Gegenuber diesen Ausführungen hat die Frage eine Berechtigung, weshalb das Gebiet der Oxoniumverbindungen erst so spät der experimentellen Bearbeitung zugänglich geworden. I)ies batte seinen Grund dariu, dass die Erkenntniss der Vierwerthigkeit des Sauerstoffs in den cyklischen Verbindungen sich erst in letzter Zeit verallgemeinert hat. Zweitens ist die Isolirung der Oxoniumsalze an gewisse Vorschriften und Bedingungen geknüft; jch möthte hier besonders die Anwendung des Eisenchlorides als Fällungsmittel hervorheben. Obgleich die Oxoniumsalze mit allen möglichen Salzen der Schwermetalle Verbindung eingehen, hat sich bis jetzt keins seiner Zugänglichkeit nach, und den Figenschaften der entstehenden Doppelsalze wegen, ebenso praktisch zu ihrer Fällung und Isolirung erwiesen. Die Eisenchloriddoppelsalze sind meistens schwer löslich, farbig, lassen sich gut krystallisiren und sind 
angenehm in der Analyse $\left.{ }^{1:}\right)$, besitzen meistens auch ziemliche scharfe Schmelzpunkte. Wir haben sie, wie aus dem experimentelien Theil zu ersehen ist, stets zur Charakterisirung henutat und haben die oxydirenden Eigenschaften des Eisenchlorides niemals störend empfunden. Immerhin wäre es dieser Lmstaud, der im Falle ron Amido- oder Oxyderivaten ein anderes Fällungsmittel empfehlenswerth erseheinen liesse, z. B. Quecksilherchlorid.

\section{Ueber die Farbe der Salze in den höheren Reihen, die Pyrylium- oder isologe Ringe enthalten.}

Die Untersuchungen haben ron den einfachsten lieihen aufsteigend zu hochmolekularen complexen Ringen, die so ziemlich an der Grenze des morphologisch bekannten Gebilde liegen, hinaufgefüht. Die Farbe der entsprechenden Verbindungen vertieft sich immer mehr, und erreicht schliesslich dic Stärke wirklicher Farbstoffe.

Es schien uns von Wichtigkeit, die auf Farbe beziglicben Angaben in den verschiedenen isologen und homologen höheren Pyryliumringen übersichtlich in einer Tabelle zusammenzustellen, da das hier geförderte Material eine wesentliche Ergänzung in der henntuiss derselben bedeutet und zugleich eiuen Ueberblick uber das ganze Gebiet, wie auch über die Nomenclatur, zu geben.

12) Ihe Verbrenunuren gelingen recht gut. Wir haben für die Eisenund Chloranalyse einen gemeinsamen Wry eingeschlagen, der sich auch in anderen Fällen für die Analyse ähulicher Eisensalze bewährt hat. Jan löst etwa $0,2 \mathrm{~g}$ Substanz in $15 \mathrm{ccm}$ Alkohol, verdinnt auf $200 \mathrm{ccm}$ mit Wasser, erhitzt etwa zwei Stunden auf dem Wasserbade unter Zusat\% vou wenigen Tropfen Salpetersäure, um die Fällung von basischen Fisensalzen zu verhiiten, filtrirt von der Base, die sich oft in Form von unlöslichen hystallen ausgeschieden hat, ab und fillt nun in der nur noch Anorganisches enthattenden lilissigkeit das Eisen mit Ammoniak und in dem Filtrate das (hlor nach den ibblichen verfahren aus. 


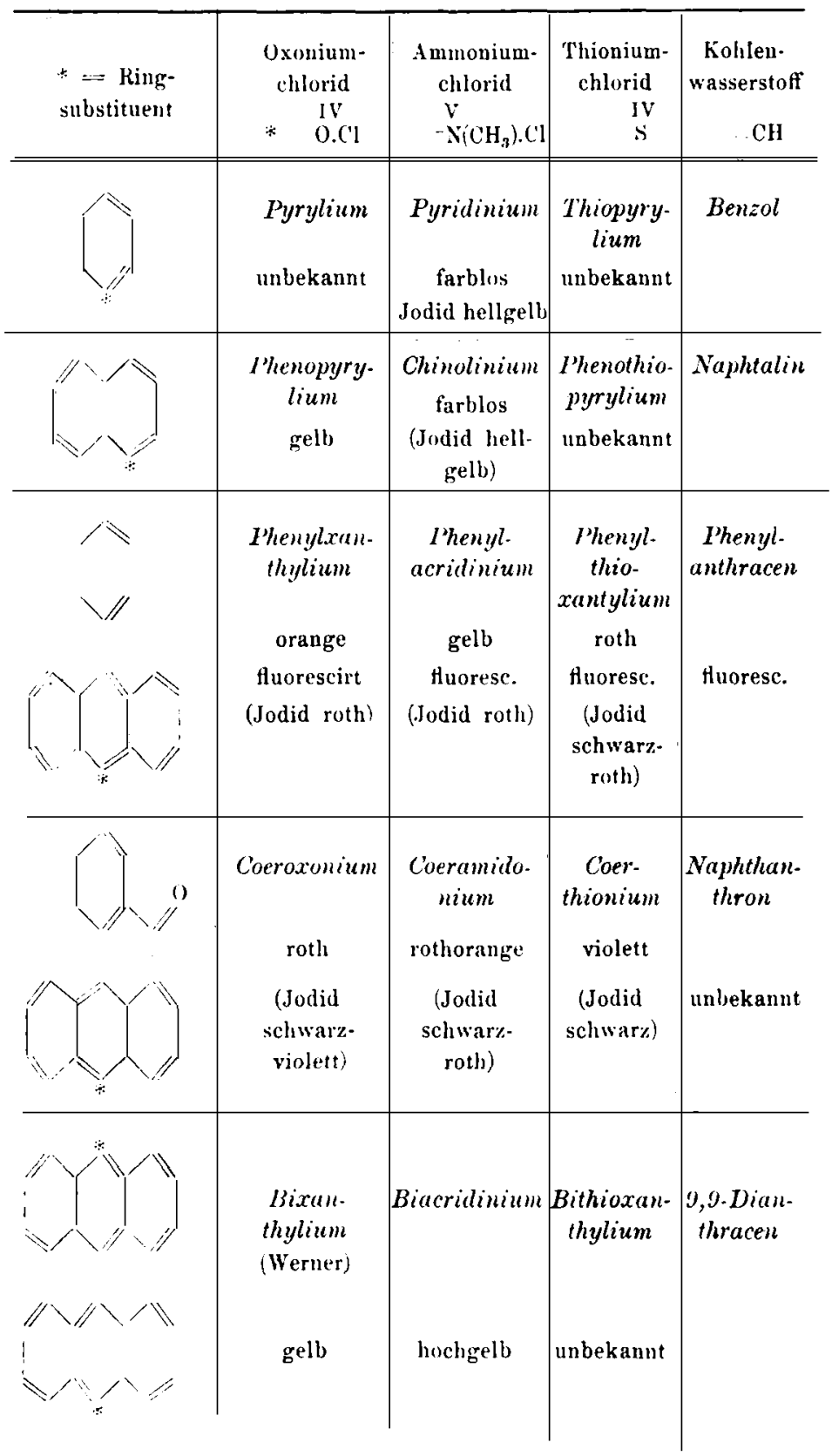




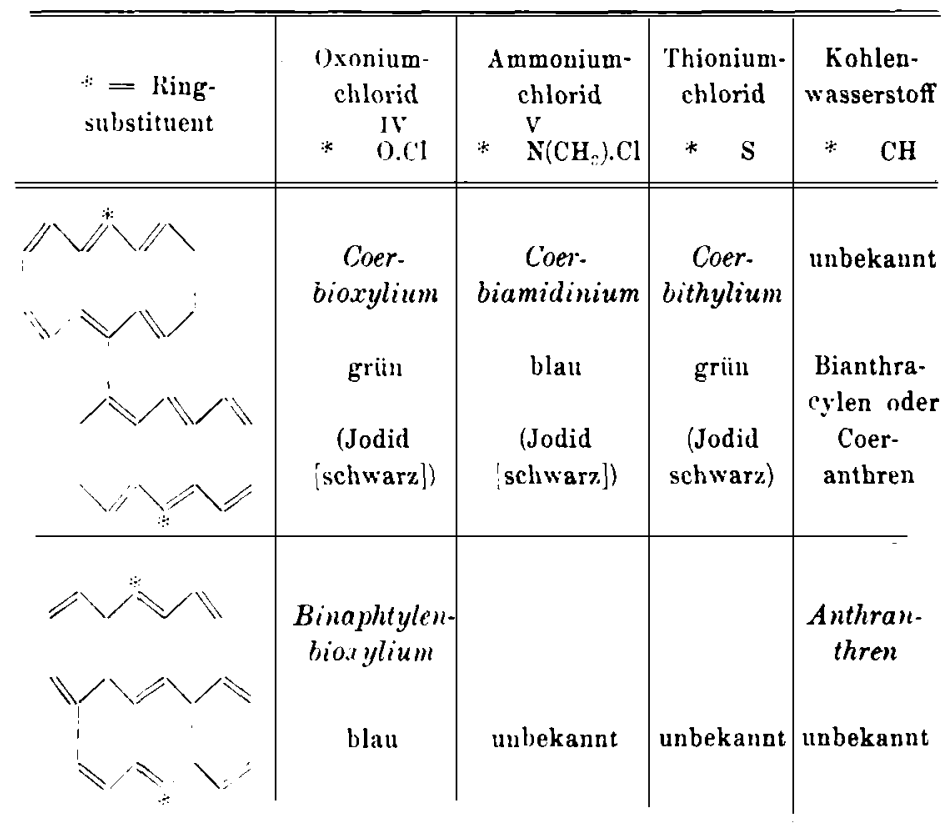

Betrachtet man die Tabelle, so ist eine erste Regelmässigkeit in den horizontalen Reihen, welche die isologen Stickstoff-, Sauerstoff- und Schwefelverbindungen nebeneinander enthalten, zu bemerken, nämlich die zunehmeude Vertiefung der Farbe, der Oxoniumverbindungen ${ }^{13}$ ) gegenuber den Ammoniumverbinllungen und den Thioniumverbindungen gegenüber ersteren: eine Regelmässigkeit, die in den Reiben der Phenazonium-, Azothionium- und Azoxoniumverbindungen wiederkehrt.

Betrachtet man die verticalen Reihen, so sieht man hier auch cine regelmässige Verstärkung und Vertiefung der Farbe mit der Anhäufung der Ringe Hand in Hand geben. In der Reihe der Coeroxoniumverbindungen wirkt ausserdem die Ketongruppe farbverstärkend und die entsprechenden Salze baben schon dic Farbstärke von wirklichen Farbstoffen.

13. Die Salze sind als (hloride angeführt. Betreffs der Farbe der Jodide vergleiche H. Decker, Ueber ionisirbare Chromophore, Ber. d. dentsch. chem. Gies. 37, 2938. 
Die Verkuppelung der Ringe, wie sie in dem Coerbioxylium (und folgenden Ringen) auftritt, bedingt ein besonders intensives Ilerwortreten der Farbe. Dieser Effect wird im hohen Grade durch die Verschweissung ${ }^{14}$ ) der linge gleichzeitig durch mehrere Bindungen hervorgerufen, wie das aus dem Vergleiche der grünen Coerbioxylium- mit den gelben Bixanthyliumsalzen hervorgeht. Diese unterscheidet sich von jenen uur durch den Mehrgehalt ron zwei Wasserstoffatomen. Das Binaphtylenbioxylium, bei dem zwei Xanthyliumringe durch drei Bindungen verschweisst sind, zeigt im Verbältnisse zu seiner liohlenstotizahl eine äusserst intensive larbe (Indigoblau).

Diese hier haun angedeuteten Verhältnisse bei den mehrkernigen complexen Ringen tinden ihr Gegenstück in den hochmolekularen Kupenfarbstoffen der Anthracenreihe. Auch dort scheint nicht die Anzabl der im Molekül stehenden Anthracenkerue, sondern ihre compacte Lagerung an einander, ihre Verschweissung, die Verticfung der Farbe zu bedingen. Natürlich sind die hier angeführten einfachsten hörper sogenannte Chromogene, d. b. die Muttersubstanzen von viel intensiveren wirklichen Farbstoffen ${ }^{15}$ ), welche letzteren erst durch den Eintritt von Amido- and Oxygruppen u. s. w. entstehen.

Es wüde uus viel zu weit führen, hier die einzelnen bekannten Fälle auch nur anzuführen. Es sei aber darauf hingewiesen, dass bereits die Oxyderivate in der Chinoliniumreihe, wo die einfachen Salze ja farblos sind, farbige Körper sind und dass die Oxyderivate in der Phenopyryliunreihe, wie aus den Litersuchungen von $B$ ül ow hervorgeht, bereits rothe Farbstoffe sind. Die Dioxyderivate des Xauthyliums sind intensiv roth. Die Oxyderivate des Phenylxanthyliums sind Farbstoffe

14) Von zwei Kürpern mit der gleichen Anzahl Atomen im skelett wird stets derjenige die tiefere Farbe anficisen, der mehr linge, also weniger Wasserstoff enthialt.

15) Theilweise von technischer Bedentung wie Rhodamin, Fluorescein, Coeruleïn, lyounin. 
der Fluoresceingruppe. Ein Polyoxyderivat aus der Coeroxoniumgruppe ist der grüne Farbstoti Coeruleïn.

\section{Kapitel I.}

\section{Phenopyryliumreihe.}

Die ersten Anzeigen von der Existenz der Phenopyryliumsalze traten uns bei der Verarbeitung des Einwirliungsproductes von Bromphenylmagnesium auf Cumarin in Form eines krystallinischen Niederschlags, den der Salzsäureauszug mit Eisenchlorid gab, entgegen. Fs gehörte aber cine Abänderung des gewöhnlichen Verfahrens bei der Grignard'schen Synthese dazu, um auf diesem Wege zur Analyse genügende Mengen des Doppelsalzes zu erhalten. Das Grignard'sche Reagens musste tropfenweise in einen Ceberschuss der Cumarinlösung hineingebracht werden. Als nun die Analysen gezeigt hatten, dass der gesuchte Körper auch wirklich vorlag, haben wir eine zweite Synthese aus Oxybenzalacetophenon ausgefulhrt und auf diesem Wege eine Methode gefunden, nach der man in kurzer Zeit beliebige Mengen von Phenopyryliumsalzen herstellen hann:



Darauf wurde die Synthese der Methyl-o-phenopyryliumsalze sowohl aus dem Methyl-o-cumarketon als auch aus dem Cumarin mit Methylmagnesiumjodid ausgefuhrt : 
296 Decker und $v$. Fellenberg, Leber Phenopyrylium

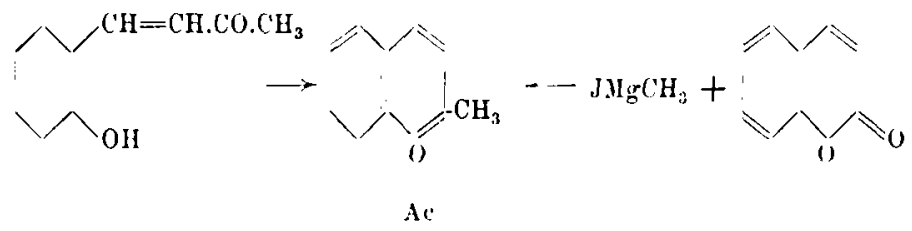

Schliesslich condensirten wir das Gluco-o-cumaraldehyd (I) und gelangten so zu den Salzen des einfachsten Phenopyryliums ${ }^{16}$ ) (II) :

I.

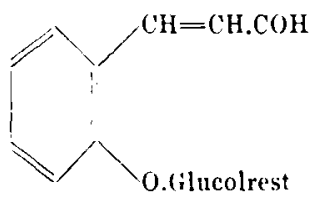

II.



Damit war endlich die Muttersubstanz der verschiedenen natürlichen und synthetischen Farbstoffe der Flavonreihe, die das Arbeitsgebiet r. Kostanecki's sind, aufgefunden worden.

Noch näher unserem Phenopyrylium stehen die Salze der „Benzopyranole“ von Bulow, die bei der Condensation von 1,3-Diketonen mit mehrfach hydroxylierten Phenolen in grosser Zabl dargestellt worden sind. Es fehlt bier an Raum, auf die Constitutionen der einzelnen Verbindungen einzugehen, doch soviel muss gesagt werden: diese Salze sind ihrer Bildung und Beschreibung nach einfach die Sulstitutionsproducte der hier

16) Zur Numerirung des Ringes wollen wir die in der isologen Chinolinreihe gebräuchliche Zahlenstellung verwenden. Flavon,

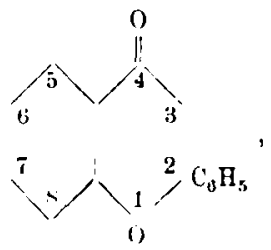

wäre nach dieser Nomenclatur Phenyl-(2-)phenopyron-( 4 ). 
beschriebeuen Phenopyryliumsalze. Was die Basen anhetrifft, ist ibre Constitution, falls man nicht eine chromophore Phenolbetainbildung (Formel I) zwischen der Hydroxylgruppe und dem vierwertigen Sauerstoff annehmen will, ebenso wie in der Chinolinreibe zu deuten, nämlich durch Annahme der Wanderung des Hydroxyls in Stellung 2 (und nicht in Stellung 4). Entsprechend der Bildung der Chinolanole $(2)$ und Pyridanole $(2)^{17}$ ) werden sich hier Phenopyranole (2) (Formel II) bilden. Die Spaltungsproducte mit Alkali stehen mit diesen neuen Formeln in bestem Einklange.

I.

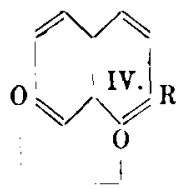

II.



Bei dieser Auffassung fällt auch die unwabrscheinliche Formel eines $\alpha$-Lactons, zu der $B u J 0 w^{18}$ ) in einem Falle geleitet worden ist, weg. Der betreffeude Körper ist rielleicht das $\gamma$-Lacton einer Benzoyloxy(7)-phenyl(2)-phenopyranol(2)carbonsaure (4). Neuerdings scheint Gault ${ }^{19}$ ) aus Salicylaldebyd und Oxalessigester auch ein Phenopyryliumsalz gewonnen zu haben, dem er allerdings eine andere Formel giebt. Was die Verbindungen aus der einfachen Pyryliumreibe anbetrifft, welche derselbe Forscher dargestellt haben will und denen diese Fähigkeit, Salze zu bilden, abgeht, so spricht letzterer Umstand eher dafür, dass die angenommene Structur

17) Es ist dem Einen von uns trotz der grossen $Z a h l$ von Chinolonen-(2), die aus den Chinolinjodmethylaten dargestellt worden sind, weder in der Pyridin- noch in der Chinolinreihe gelungen, 4-Derivate zu beobachten. Das Phenyl-(2-iphenopyron-(4) (Flavon) (Ber. d. d. chem. (ies. 36, 1949) als Oxydationsproduct wiirde ja für die (4-Stellung) der Hydroxylgruppe sprechen. Doch ist zu berücksichtigen, dass die Oxydation in saurer Iösuner ausgefuhrt wurde.

14) B ii low, Ber. d. deutsch. chen. Ges. s6, 1949.

19) (i a ult, Bulletins 36, 1273 (1906. 
von Pyranderivaten diesen hörpern, entgegen der Auftassung ihres Eutdeckers, nicht zukommt. Knoevenagel ${ }^{20}$ ) hat aus dem Condensationsproducte von Salicylaldebyd und substituirtem Acetylaceton mit Salzsäure eine gelbe Lösung erhalten, die ohne Zweifel ein Substitutionsproduct des Phenopyryliumchlorids enthielt.

\section{Salze des Phenopyryliums,}



Salicin geht durch Oxydation mit Salpetersäure, nach Schiff (diese Aunalen 1.j4, 19), in Helicin über. Dieses reagiert, nach Tiemann und kees (Ber. d. deutsch. chem. Ges. 18, 1958), mit Acetaldehyd unter Bildung von Gluco-o-cumaraldehyd.

Frisch dargestellter, womöglich noch feuchter, oder aber fein pulverisirter Gluco-0-cumaraldehyd wird einige Zeit mit concentrirter Salzsäure erhitzt; heftige Kohlendioxydentwickelung und Schaumbildung tritt ein. Man verdünnt mit dem gleichen Volumen 1j-procentiger Salzsäure, küblt $a b$ und filtrirt von einem ausgeschiedenen, schwarzen Körper ab. Die Lösung enthält nun das leicht lösliche Phenopyryliumchlorid.

Das Eisenchloriddoppelsalz wird aus dieser Lösung mit festem Eisenchlorid ausgefällt und als gelbes, krystallinisches Pulver rom Scbmelzp. $199^{\circ}$ (corrigirt) erhalten. In Salzsäure ist es sehr schwer löslich, etwas leichter in Eisessig.

Die Analyse wurde, nach dem Trocknen bis zu constantem Gewicht im Exsiccator, ausgeführt.

$0,1994 \mathrm{~g}$ gaben $0,2395 \mathrm{CO}_{3}$ und $0,0421 \mathrm{H}_{\mathrm{g}} \mathrm{O}$.

$0,2052_{\mathrm{r}}^{\mathrm{r}}, \quad, \quad 0,0496 \mathrm{Fe}_{2} \mathrm{O}_{3}$ und $0,350: 3 \mathrm{AgCl}$.

20) K noevenagel, Ber. d. deutsch. chem. (ies. 37, 4494. 
und höhere homologe und isologe Pyryliumringe. $\quad 299$

$\begin{array}{lcr} & \text { Berechnet fuir } & \text { Gefunden } \\ & \mathrm{C}_{9} \mathrm{H}_{7} \mathrm{OClFeCl} ; & \\ \text { c } & 32,84 & 32,76 \\ \mathrm{H} & 2,15 & 2,36 \\ \mathrm{Cl} & 43,12 & 42,21 \\ \mathrm{Fe} & 17,113 & 16,92\end{array}$

Wird das Eisensalz in wenig Aceton gelöst, mit viel Wasser versetzt und erhitzt (wie es zur Bestimmung des Fisens und Chlors geschicht), so riecht diese Lösung intensiv nach Fenchel. Das entsprechende Metbylderivat liefert bei dieser Behandlung einen ähnlichen, jedoch nicht definirbaren Geruch.

Das Goldchloriddoppelsalz fällt aus der Oxoniumchloridlösung in bräunlichgelben, mikroskopischen Nadeln vom Schmelzpunkt $198-200^{\circ}$ (corrigirt) aus. Nach dem Trocknen im Exsiccator bis zu constantem Gewicht wird es analysirt.

0,1455 g gabeu $0,1210 \mathrm{CO}_{\mathrm{g}}, 0,0206 \mathrm{H}_{2} \mathrm{O}$ und $0,0613 \mathrm{Au}$.

$\begin{array}{lcr} & \text { Berechnet für } & \text { ('iefunden } \\ & \mathrm{C}_{9} \mathrm{H}_{7} \mathrm{OCl}\left(\mathrm{AuCl}_{3}\right) & \\ \mathrm{C} & 23,01 & 22,67 \\ \mathrm{H} & 1,50 & 1,58 \\ \mathrm{Au} & 42,02 & 42,10\end{array}$

5. Salze des Methyl(2)-phenopyryliums,<smiles>C=CCC=C</smiles>

Als Ausgangsproduct dient Methyl-o-cumarketon, welches nach Harries (Ber. d. deutsch. chem. Ges. 24, 3180) durch Condensation von Salicylaldehyd mit Aceton dargestellt wird. Derselbe wird mit concentrirter Salzsäure einige Minuten gekocht, mit 15-procentiger Salzsäure zur Hälfte verdünnt, von einem ausgeschiedenen rothen Farbstoff abfiltrirt und das Filtrat mit festem Eisenchlorid fractionirt gefällt. Zuerst fallen dunkel gefärbte, harzige Verunreinigungen aus und bei weiterem Zu- 
satze erhält man das Eisenchloriddoppelsalz in hellgelben, kleinen Nädelchen rom Schmelzp. $118-119^{\circ}$ (corrigirt).

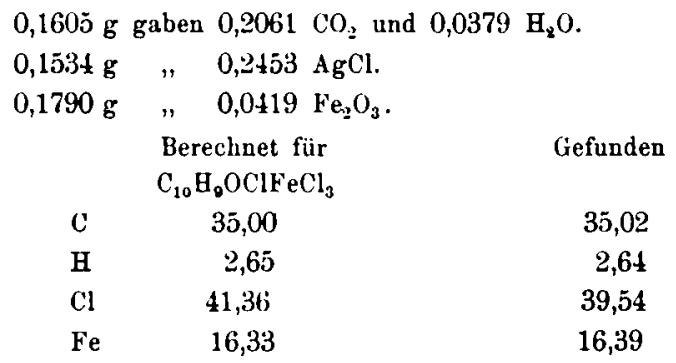

Das Cadmiumbromiddoppeisalz wird als gelbes Krystallpulver aus einer Oxoniumbromidlösung erhalten.

$0,1759 \mathrm{~g}$ gaben $0,1674 \mathrm{CO}_{2}$ und $0,0350 \mathrm{H}_{2} \mathrm{O}$.

$\begin{array}{ccr} & \text { Berechnet für } & \text { Gefunden } \\ & \mathrm{C}_{10} \mathrm{H}_{\mathbf{9}} \mathrm{OBr} \cdot \mathrm{CdBr}_{\mathbf{3}} & \\ \mathrm{C} & 24,14 & 25,95 \\ \mathrm{H} & 1,82 & 2,23\end{array}$

Die Carbinolbase, die man mit Wasser oder Alkalien aus den Methylphenopyryliumsalzlösungen erhält, ist äusserst leicht veränderlich und geht leicht in einen Farbstoff über. Dies darf nicht befremden, denn in der isologen Chinaldinreibe liegen die Verbältnisse ganz ähnlich und aus den quaternären Chinaldinsalzen hat man bis beute noch keine einheitliche Base mit Sicherheit isoliren können. Auch die Farbstoffbildung (Cyanine) findet dort mit derselben Leichtigkeit statt.

Einwirkung von Methylmagnesiumjodid auf Cumarin. In eine erwärmte Lösung von Cumarin in getrocknetem Benzol wird $1 / 2$ Mol. Methylmagnesiumjodid tropfenweise einfliessen gelassen; eine ölige Schicht scheidet sich ab. Nach Abdestilliren des Lösungsmittels im Marienbade bleibt eine gelbe Masse zurück, die mit 1j-procentiger Salzsäure geschuttelt wird, wobei sie zum Teil in Lösung geht. Beim Versetzen der salzsauren Lösung mit festem Eisenchlorid krystallisiren grünlichbraune Nädelchen von metallischem Schiller aus. Nach Umkrystallisiren aus Eisessig und Salzsäure schmelzen dieselben 
bei 56-60 6 . Die qualitative Analyse zeigt, dass hier ein Jodid vorliegt. Dasselbe ist nicht beständig und verliert constant Jod. Nach 24-stlundigem Stehen uber Natron und rothem Phosphor wurden folgende Zablen erhalten:

$0,1646 \mathrm{~g}$ gaben $0,2565 \mathrm{CO}_{2}$ und $0,0546 \mathrm{H} \_$.

$\begin{array}{ccc} & \text { Berechnet für } & \text { Gefunden } \\ & \mathrm{C}_{10} \mathrm{H}_{9} \mathrm{OJ} & \\ \mathrm{C} & 44,13 & 42,50 \\ \mathrm{H} & 3,34 & 3,71\end{array}$

Der Körper wurde noch eine Woche lang im selben Exsiccator gelassen und wieder analysirt. Der Schmelzpunkt stieg inzwischen auf $68-70^{\circ}$ und die Analyse ergab ein Ansteigen des Kohlenstoffgehaltes um $6 \mathrm{pC}$. Wird das Salz mit Wasser und etwas schwefliger Säure gekocht, so geht es theilweise in Lösung und nach dem Erkalten krystallisirt Cumarin aus. Ein ähnliches Verbalten der Chinaldinderivate hat der Eine von uns mit Remfry bei dem Nitrochinaldinjodmetbylat constatirt, welches unter dem Einflusse von Oxydationsmitteln in Nitrometbylchinolone überging. Die aus dem Methylcumarketon gewonnene Oxonium-Chloridlösung geht durch Zusatz von Jodkalium in ein Salz von den gleichen Eigenschaften uber. $\mathrm{Da}$ uns einerseits die Synthese aus Cumarin gegenuber der anderen nur Sachtheile bot und andererseits der Verlauf der Grignard'schen Reaction an dem gleich im Folgenden beschriebenen Beispiele genügend aufgeklärt erschien, haben wir diesen Gegenstand nicht weiter verfolgt.

\section{Derivate des Phenyl(2)-phenopyryliums,}

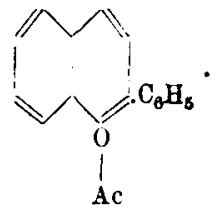

Darstellung aus $O x y(2)$-benzalacetophenon. Oxybenzalacetophenon wird nach $\mathrm{Bablich}$ und $\mathrm{K}$ ostanecki (Ber. d. deutsch. chem. Ges. 29, 233) durch Condensation von Salicylaldebyd mit 


\section{Decker und v. Fellenberg, Ueber Phenopyrylium}

Acetophenon dargestellt. Es condensirt sich zu Phenylphenopyryliumchlorid sowobl durch Sättigen der ätherischen oder alkobolischen Lösung mit Salzsäuregas, als auch durch einfaches Erhitzen mit concentrirter Salzsäure mit oder ohne Eisessigzusatz. Wir haben uns aber in diesem Falle nicht begnügt, die Existenz des Oxoniumchlorides in Lösung durch die Fällung einer Anzabl weiter unten beschriebener Doppelsalze zu constatiren, sondern auch das einfache Chlorid in Substanz dargestellt.

Chlorid, $\mathrm{C}_{15} \mathrm{H}_{11}$ O.Cl. Oxybenzalacetophenon (gut getrocknetes) wird in über Natrium getrocknetem Aether gelöst und in die Lösung trocknes Salzsäuregas bis zur Sättigung eingeleitet. Nach dem Erkalten krystallisirt das Cblorid in hellgelben, glitzernden Blättchen aus. Der Schmelzpunkt liegt bei $69-70^{\circ}$ (corrigirt), wenn einige Krystalle der Lösung entnommen, auf Thon gepresst, sogleich ins Schmelzröhrchen gebracht und rasch erhitzt werden. Der Körper ist bygroskopisch; uberlässt man ihn sich selbst an der Luft, so beginnt er schon nach einigen Secunden in Folge von Wasseranziehung und Zersetzung zu sintern, deshalb wurde von einer Analyse abgeseben.

Um die Geschwindigkeit der Condensation mit Salzsăuregas auch in der Kälte festzustellen, wurden $5 \mathrm{~g}$ Oxybenzalacetophenon in $25-30 \mathrm{ccm}$ Alkohol gelöst und unter Kublung Salzsăure bis zur Sättigung eingeleitet. Beim Verdunnen mit Wasser und Zusetzen von Natronlauge tritt die orangerothe Färbung des Oxybenzalacetophenonnatriumsalzes in geringem Maasse auf, obne dass jedoch mit Säure ein Niederschlag dieses Körpers entsteht. Bis auf Spuren ist also das Ausgangsmaterial in etwa zebn Minuten verändert worden.

Aus der.so gewonnenen alkoholisch salzsauren Lösung in frischem Zustande können Phenylphenopyryliumdoppelsalze dargestellt werden. Die Lösung verändert sich jedoch ziemlich rasch beim Stehen, so dass die Salze schon am nächsten Tage unrein ausfallen.

Eine bessere Darstellung der Salze ist folgende: Oxybenzalacetophenon wird in wenig rauchender Salzsäure unter 
und höhere homologe und isologe Pyryliumringe. 303

Zusatz von $1 / 5$ des Volumens Eisessig gelöst und einige Minuten zum Sieden erhitzt. Darauf wird das Metallchlorid in Ueberschuss fest oder in concentrirter Lösung zugefügt. Das Doppelsalz fällt als dicker Krystallbrei aus, welcher sich in der Kälte noch bedeutend vermehrt.

Das Eisenchloriddoppelsalz. Dieses am leichtesten zu erhaltende Salz kann auch folgendermassen dargestellt werden: Oxybenzalacetophenon wird in Alkohol gelöst, in die Lösung viel festes Eisenchlorid eingetragen, auf dem Wasserbade erhitzt und unter allmählicher Kühlung Salzsäuregas eingeleitet, bis die Lösung in der Kälte damit gesättigt ist. Ein dicker Brei des Doppelsalzes fällt aus. Nach dem Umkrystallisiren aus Eisessig zeigt dasselbe den Schmelzp. $125-129^{\circ}$ (corrigirt).

Ein Teil wird bis zum constanten (iewicht im Exsiccator getrocknet und verbrannt.

$0,2003 \mathrm{~g}$ gaben $0,3274 \mathrm{CO}_{2}$ und $0,0542 \mathrm{H}_{2} \mathrm{O}$.

$\begin{array}{ccc} & \text { Berechnet für } & \text { Gefunden } \\ & \mathrm{C}_{15} \mathrm{H}_{\mathbf{1 1}} \mathrm{OCl}_{\mathrm{FeCl}} & \\ \mathrm{C} & \mathbf{4 4 , 4 6} & \mathbf{4 4 , 5 7} \\ \mathrm{H} & 2,74 & 3,03\end{array}$

Das Goldchloriddoppelsalz bildet braungelbe Kryställchen, die nach vorhergehendem Sintern bei $200-202^{\circ}$ schmelzen. Der Körper wird bis zu constantem Gewicht im Exsiccator getrocknet und analysirt.

$0,1657 \mathrm{~g}$ gaben $0,1912 \mathrm{CO}_{2}, 0,0355 \mathrm{H}_{2} \mathrm{O}$ und 0,0599 Au.

$\begin{array}{lcr} & \text { Berechnet fiür } & \text { (iefunden } \\ & \mathrm{C}_{15} \mathrm{H}_{11} \text { OCl.AuCl } & \\ \mathrm{C} & 32,96 & 31,47 \\ \mathrm{H} & 2,03 & 2,40 \\ \mathrm{All} & 36,11 & 36,15\end{array}$

Das Quecksilberchloriddoppelsalz bildet braungelbe Nadeln vom Schmelzp. $183-185^{\circ}$

Das Cadmiumbromiddoppelsalz, aus einer bromwasserstoffsauren Lösung des Phenylphenopyryliums gefällt, bildet braun- 


\section{Decker und $v$. Fellenberg, Ueber Phenopyrylium}

gelbe Nädelchen, die nach vorbergehendem Sintern bei $190^{\circ}$ bis $192^{\circ}$ schmelzen.

$0,1932 \mathrm{~g}$ gaben $0,1758 \mathrm{AgBr}$.

$$
\begin{array}{ccc} 
& \text { Berechnet für } & \text { Gefunden } \\
& C_{15} \mathrm{H}_{13} \mathrm{OBr} \cdot \mathrm{CdBr} r_{2} & \\
\text { Br } & 42,91 & 39,78
\end{array}
$$

Das Perbromid, $\mathrm{C}_{15} \mathrm{H}_{11} \mathrm{OBr} .\left(\mathrm{Br}_{2}\right)$, ist äusserst schwerlőslich und fällt aus sehr verdunnten Lösungen aus. Es wird dargestellt durch Lösen von Oxybenzalacetophenon in concentrirter Bromwasserstoffsäure unter Zusatz von etwas Eisessig und Versetzen der Lösung mit Brom. Das feinkrystallisirte, orangeroth gefärbte Product schmilzt bei $122^{\circ}$ (corrigirt). Es wird uber festem Natronhydrat bis zum constanten Gewicht getrocknet und analysirt.

$0,2575 \mathrm{~g}$ gaben $0,3751 \mathrm{CO}_{\mathrm{a}}$ und $0,0556 \mathrm{H}_{\mathrm{a}} \mathrm{O}$.

$0,2002 \mathrm{~g} \quad, \quad 0,2495 \mathrm{AgBr}$.

$\begin{array}{lcr} & \text { Berechnet für } & \text { Gefunden } \\ & \mathrm{C}_{13} \mathrm{~B}_{11} \mathrm{OBr}\left(\mathrm{Br}_{2}\right) & \\ \mathrm{C} & \mathbf{4 0 , 2 7} & \mathbf{3 9 , 7 3} \\ \mathrm{H} & 2, \mathbf{4 8} & \mathbf{2 , 4 2} \\ \mathrm{Br} & 53,67 & \mathbf{3 3 , 0 4}\end{array}$

Das Perjodid, dem aller Wabrscheinlichkeit nach die entsprechende Formel $\mathrm{C}_{15} \mathrm{H}_{11} \mathrm{OJ}\left(\mathrm{J}_{2}\right)$ zukommt, wird durch Fällen mit jodhaltiger Jodwasserstoffsăure und Verdünnen mit Salzsäure dargestellt. Es besitzt die Farbe des rothen Phosphors, riecht nach Jod und schmilzt nach vorbergebendem Sintern bei $147-148^{\circ}$.

$0,1322 \mathrm{~g}$ gaben $0,1557 \mathrm{CO}_{2}$ und $0,0254 \mathrm{H}_{2} \mathrm{O}$.

$$
\begin{array}{ccc} 
& \text { Berechnet für } & \text { Gefunden } \\
& \mathrm{C}_{15} \mathrm{H}_{11} \text { OJ. }\left(\mathrm{J}_{\mathbf{2}}\right) & \\
\mathrm{C} & 30,63 & 32,12 \\
\mathrm{H} & 1,89 & 2,15
\end{array}
$$

Das Jodid erbält man als orangerothe Kryställchen, wenn man eine concentrirte Lösung von Chlorid in Eisessig mit Jodkalium in der Wärme versetzt, nach dem Erkalten. Es 
und höhere homologe und isologe Pyryliumringe. 305

ist etwas tiefer gefärbt als das Jodmetbylat des $\alpha$-Phenylchinolins, welches gelb ist. Es ist ziemlich leicht löslich.

Phenylphenopyryliumsalze aus Cumarin. Eive erwärmte Lösung von $10 \mathrm{~g}$ Cumarin in $60 \mathrm{ccm}$ Aether wird unter Anwendung von Druckluft tropfenweise mit $1 / 2$ Mol. einer ätherischen Lösung von Magnesiumbrombenzol versetzt. Jeder Tropfen erzeugt sofort einen gelben Niederschlag, die Lösung gerăth in heftiges Sieden. Zum Schlusse wird der Aether im Wasserbade abdestillirt, wobei das gelbe Product zu einer dickflüssigen, orange gefärbten Masse zusammenscbmilzt. Dieselbe wird mit viel Wasser geschüttelt, abfiltrirt und so das Magnesium zum grossen Theile entfernt. Der Ruckstand wird mit $35 \mathrm{ccm}$ rauchender $\mathrm{HCl}$ unter Erhitzen kräftig geschüttelt und nach dem Erkalten vom harzigen Ruckstande, der zum Theil aus unverändertem Cumarin besteht, abfiltrirt. Die so erbaltene salzsaure Lösung wird mit etwas Eisessig geklärt und mit festem Eisenchlorid in grossen Ueberschuss versetzt.

Das Phenyl(2)-phenopyryliumchlorideisenchloriddoppelsale fallt in glänzenden, gelben Nadeln aus; umkrystallisirt aus Eisessig schmelzen dieselben nach vorhergehendem Sintern bei $126-128^{\circ}$ (corrigirt). Das Salz ist in jeder Beziehung mit dem aus Salicylaldebyd dargestellten und oben beschriebenen identisch. Das Präparat wurde im Exsiccator bis zu constantem Gewicht getrocknet und analysirt.

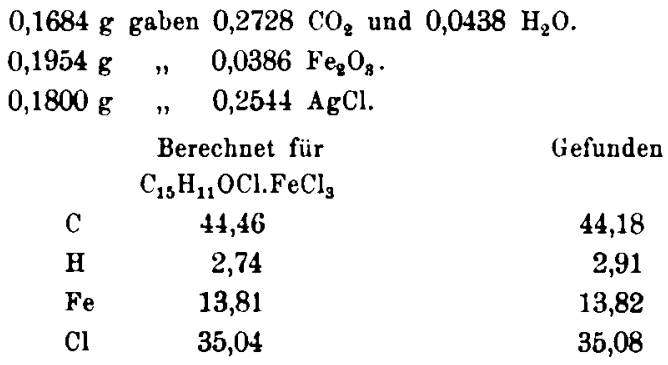

Auf analoge Weise wird das Phenylphenopyryliumchloridquecksilberchlorid in langen, bräunlicbgelben Nadeln vom 
Scumelzp. $183-185^{\circ}$ erbalten. Auch dieses Salz ist dem nacb der anderen Synthese dargestellten identisch.

Ueber ein in Salzsäure unlösliches, höher schmelzendes Nebenproduct dieser Reaction, das ebenfalls aus Cumarin entstebt und die Analysen auf ein Diphenylpyran stimmen, wird später berichtet werden.

J. Houben ${ }^{21}$ ) hat die Einwirkung von Magnesiumbalogenalkylen auf Cumarin bereits untersucht und erhielt unter anderen Versuchsbedingungen abweichende Resultate. Wir sind ubereingekommen, die verschiedenen Producte gemeinsam zu studiren, um ihre Beziehungen unter einander aufzulkären.

Phenyl(2)-phenopyranol(2) (Carbinolbase des Oxoniumsalzes),



Die direct bei der Synthese erhaltene alkoholische Lösung des Chlorides wird in viel Wasser gegossen und mit Natronlauge etwas abgestumpft. Ein grünlicbbrauner, krystallinischer Körper fällt aus. Derselbe wird abfiltrirt, in Salzsäure gelöst, durch Ausschütteln mit etwas Benzol gewisse Verunreinigungen entfernt und mit festem Eisenchiorid das Doppelsaiz ausgefält. Nach dem Umkrystallisiren aus Eisessig zeigt es den richtigen Schmelzpunkt, Dadurch ist der Beweis geliefert, dass die Carbinolbase in dem Niederschlage vorhanden war. Es gelingt jedoch nicht, sie durch Umkrystallisiren zu reinigen, da sie $z u$ veränderlich ist. Auch bei dem isologen, zu diesem Zwecke dargestellten Phenyl(?)chinolinjodmethylat konnte der Eine von uns das entsprechende Chinolanol vicht im analysepreinen Zustande erbalten.

Beim Kochen einer sehr kleinen Probe von Quecksilberchloriddoppelsalz mit Wasser ging es in Lösung. Beim Abküblen scheidet sich die Base in ziemlich reinem Zustande

2) Ber. d deutsch. chem. Ges. 37, 489, 502. 
$a b$; sie giebt ohne Weiteres ein Eisensalz von reiner Farbe und richtigem Schmelzpunkt. Wird der Versuch jedoch mit einer etwas grösseren Menge $(2 \mathrm{~g})$ Quecksilbersalz angestellt, 80 wird nur ein harziges Product erbalten, da offenbar das Anwärmen der Lösung zu lange dauert.

Wird das in ätherischer Lösung dargestellte Phenyl(2)phenopyryliumchlorid mit $\mathrm{NH}_{3}$ ubergossen, mit Wasser ausgewaschen und das so erhaltene dickflüssige und gefärbte Product im Schiffchen bei $130^{\circ}$ getrocknet und verbrannt, so erhält man auf das Phenylphenopyranol stimmende Zahlen.

$0,1247 \mathrm{~g}$ gaben $0,3658 \mathrm{CO}_{2}$ und $0,0585 \mathrm{H}_{2} \mathrm{O}$.

$\begin{array}{lcr} & \text { Berechnet für } & \text { Gefunden } \\ & \mathrm{C}_{15} \mathrm{H}_{\mathbf{1 2}} \mathrm{O}_{\mathbf{2}} & \\ \mathrm{C} & 80,36 & 80,01 \\ \mathrm{H} & 5,40 & 5,25\end{array}$

Kapitel II.

Xanthyliumreihe.

Die Einwirkung von Phenylmagnesiumbromid auf Xanthon führte Bunzly ${ }^{22}$ ) und Decker zu den (9)-Phenylxanthyliumsalzen. Diese Synthese ist später durch v. Fellenberg ${ }^{23}$ ) fur die Darstellung von (9)-Methyl- und (9)-Benzylderivaten angewandt worden.

Hier haben wir (9)-Tolylderivate aus Xanthon und 0-Bromtoluol erhalten und zweitens aus zwei isomeren Methoxylxanthonen und Brombenzol isomere Methoxylphenylxanthyliumsalze dargestellt. Weiter sind zwei Eisendoppelsalze ron Fluoran und Dimetbylfuoran beschrieben, die zum Vergleiche mit den übrigen Oxoniumdoppelsalzen dargestellt wurden. Unseren heutigen Anschauungen nach sind es Salze der Xanthyliumcarbonsäure. Derartige Salze sind ja der Ausgangspunkt einer Betrachtung

29) Ber. d. deutsch. chem. Ges. 87, 2932.

$\left.{ }^{23}\right)$ Ber. d. deutsch. chem. Fes. 38, 2494. 
gewesen, von der aus $\mathrm{Hewitt}$ die Anwesenbeit des vierwerthigen Sauerstoffes in den Xanthyliumverbindungen zuerst erkannte und formulirte, und so den Grundstein zu der bier befolgten Theorie legte.

7. Derivate des o-Tolylxanthens (Formel I); mitbearbeitet von Fritz Dinner.

Es sollte die Synthese des Coeroxens (Formel II) von einem 0 -Tolylxanthen ausgehend versucht werden,

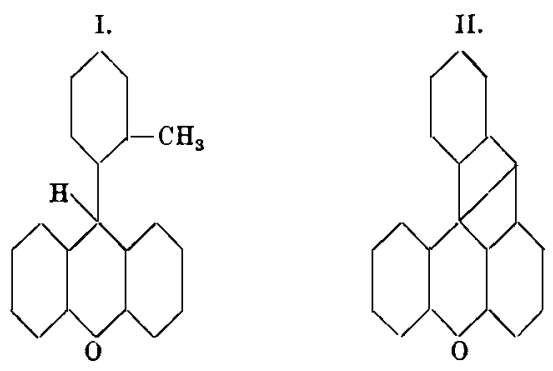

analog der Synthese des Anthracens aus o-Benzyltoluol. Die vorläufigen Versuche, das Xanthen oder die Carbinolbase durch Erhitzen oder Durchleiten durch ein erbitztes Rohr zu erhalten, haben noch keine deutlichen Resultate ergeben. Im Folgenden sind nun die bei dieser Gelegenheit aus Xanthon und Magnesiumbromtoluol gewonnenen Verbindungen beschrieben worden. o-Tolylxanthenol-(9) (Carbinolbase des Tolylxanthyliums),<smiles>CC1CCCCC1C1(O)C2CCCCC2C(=O)C2CCCCC21</smiles>

o-Bromtoluol, aus o-Toluidin dargestellt, wird in die Magnesiumverbindung, was unter sturmischer Reaction sich voll- 
und höhere homologe und isologe Pyryliumringe. $\quad 309$

zieht, verwandelt, und auf diese Xanthone einwirken gelassen. Das normale Product der Reaction wird nach dem Abdestilliren des zur Lösung gebrauchten Aethers und Benzols durch Auflösen der Reactionsmasse in Eisessig und Versetzen mit wenig Salzsäure und viel festem Eisenchlorid als Eisendoppelsalz der Oxoniumbase in Form einer krystallinischen, olivbraunen Masse isolirt.

Dieselbe kann durch Waschen mit warmem Eisessig gereinigt und dann durch Lösen in wenig Aceton und Fällen mit der sechsfachen Menge Wasser daraus die Carbinolbase gewonnen werden. Aus Benzol krystallisirt die Base in feinen, farblosen Nädelchen, die sich zu warzenförmigen Aggregaten vereinigen. Die Krystalle enthalten $1 / 2 \mathrm{Mol}$. Benzol, das bei $110^{\circ}$ fortgeht, um undurchsichtige Pseudokrystalle vom Schmelzpunkt $150,5^{0} \mathrm{zu}$ hinterlassen.

$1,0224 \mathrm{~g}$ verlieren, bei $110^{\circ}$ bis zum constanten Gewicht erhitzt, $0,1289 \mathrm{~g}$ Benzol.



Eisendoppelsalz des o-Tolylxanthyliums,<smiles>CC1CCCCC1C1C2CCCCC2C2CCCCC2C1O[Z10](C)(C)C</smiles>

Dieses Salz erbält man analysenrein, wenn man das oben bc- 
schriebene Rohsalz fortgesetzt mit warmem Eisessig digerirt, in Form eines gelben, krystallinischen Pulvers. Es schmilzt bei $208-209^{\circ}$. Aus Eisessig unter Zusatz von etwas Eisenchlorid lässt es sich $2 \mathfrak{u}$ feinglänzenden, gelbbraunen Nädelchen rom gleichen Schmelzpunkt umkrystallisiren. In seinen Eigenschaften gleicht es durchaus dem früber von Bünzly und Decker beschriebenen Eisendoppelsalz des Phenylxanthyliums.

\begin{tabular}{ccc}
$0,1824 \mathrm{~g}$ gaben $0,3418 \mathrm{CO}_{2}$ und $0,0531 \mathrm{H}_{2} \mathrm{O}$. \\
$0,2229 \mathrm{~g} \quad 0,0383 \mathrm{Fe}_{8} \mathrm{O}_{3}$ und $0,2706 \mathrm{AgCl}$. \\
\multicolumn{4}{c}{ Berechnet für } \\
$\mathrm{C}$ & $\mathrm{C}_{20} \mathrm{H}_{15} \mathrm{OCl}\left(\mathrm{FeCl}_{\mathrm{s}}\right)$ & \\
$\mathrm{H}$ & 51,18 & 51,12 \\
$\mathrm{Fe}$ & 3,22 & 3,26 \\
$\mathrm{Cl}$ & 11,94 & 12,03 \\
& 30,24 & 30,03
\end{tabular}

Nebenproducte der Grignard'schen Reaction.

Das o-Tolylxanthenol scheint nicht die einzige Substanz zu sein, welche bei dieser synthese sich bildet. Zieht man das Rohproduct der (i rignard'schen Reaction mit Petroläther aus, so erhält man aus diesem einen Körper vom Schmelzp. 16: $\geq^{\circ}$, der sich durch Umkrystallisiren aus Benzol oder Aether nicht verändert. Sowohl der Schmelæpunkt als auch der Lmstand, dass der Körper mit Benzol keine Krystallverbindung eingeht, sondern daraus ohne Veränderung in grossen Tafeln krystallisirt, lassen mit Sicherheit erkennen, dass es sich hier nicht etwa um o-Tolylxanthenol handeln kann.

Zwei Analysen, die wir machten, stimmen gut mit einander iiberein, aber die Formel und Constitution können nur weitere Lntersuchungen entscheiden.

$0,1813 \mathrm{~g}$ gaben $0,5589 \mathrm{CO}_{2}$ und $0,0944 \mathrm{H}_{2} \mathrm{O}$.

$0,1517 \mathrm{~g} \quad, \quad 0,4686 \mathrm{CO}_{2}, 0,0788 \mathrm{H} . .0$.

\begin{tabular}{cccccc} 
& \multicolumn{2}{c}{ Berechnet für } & & \multicolumn{2}{c}{ Gefunden } \\
\cline { 2 - 3 } & $\mathrm{C}_{23} \mathrm{H}_{19} \mathrm{O}_{2 .}$ & $\mathrm{C}_{28} \mathrm{H}_{20} \mathrm{O}_{2}$ & & I. & II. \\
C & 84,37 & 84,18 & & 84,07 & 84,24 \\
H & 5,85 & 6,10 & & 5,82 & 5,81
\end{tabular}

In den Mutterlaugen findet man noch glitzernde Blittchen einer Verbindung vom Schmelzp. 170".

Ausserdem findet sich in dem Rohproducte der Grignard'schen Reaction ein hochschmelzender Kohlencasserstoff, dessen Bildung ebenfalls 
zur eingehenden Untersuchung auffordert. Man erhält ihn, wenn man• das Rohproduct in Eisessig umkrystallisirt, als weniger löslichen Theil. Es muss jedoch in recht concentrirten Lösungen gearbeitet werden. Er scheidet sich in schönen Krystallen vom Schmelzp. $214^{0}$ aus. In Benzol ist er sehr !oslich und wird deshalb aus Alkohol krystallisirt.

$0,1352 \mathrm{~g}$ gaben $0,4599 \mathrm{CO}_{3}$ und $0,0822 \mathrm{H}_{2} \mathrm{O}$.

\begin{tabular}{lcccc} 
& \multicolumn{3}{c}{ Berechnet für } & Gefunden \\
\cline { 2 - 4 } & $\left(\mathrm{C}_{14} \mathrm{H}_{14}\right)_{\mathrm{n}}$ & $\left(\mathrm{C}_{\mathrm{8}} \mathrm{H}_{8}\right)_{\mathrm{n}}$ & $\left(\mathrm{C}_{14} \mathrm{H}_{13}\right)_{\mathrm{n}}$ & \\
$\mathrm{C}$ & 92,30 & 93,06 & 92,70 & 92,70 \\
$\mathrm{H}$ & 7,77 & 6,95 & 7,20 & 6,80
\end{tabular}

Danach scheint dieses Product am wahrscheinlichsten durch Zusammentreten von vier Bromtoluolresten entstanden zu sein unter Austritt von zwei At. Brom und zwei Mol. Bromwasserstoffsäure.<smiles>CC1CCCCC1</smiles>

o. Tolylxanthen,<smiles>O=C1CCCCC1C1CCCCC1</smiles>

Das Tolylxanthenol lässt sich leicht in kochender Essigsäureanhydridlösung mittelst Jodwasserstoffsäure und etwas rothem Phosphor reduciren. Beim Erkalten krystallisirt das Xanthen in Nadeln aus und wird aus Alkohol dreimal bis zum constanten Schmelzp. $119^{\circ}$ umkrystallisirt. Farblose Nädelchen.

$0,1604 \mathrm{~g}$ gaben $0,5213 \mathrm{CO}_{\mathrm{z}}$ und $0,0850 \mathrm{H}_{2} \mathrm{O}$.

Berechnet für $\mathrm{C}_{20} \mathrm{H}_{16} \mathrm{O}$

C 88,20

H $\quad \tilde{5}, 93$
Gefunden

88,64

$\overline{5}, 93$

In Schwefelsäure löst es sich in der Kälte kaum auf, in der Wärme findet lösung unter Färbung und Entstehung des Oxoniumsalzes statt, genau wie bei dem früher von uns untersuchten Benzylxanthen. 
8. Methoxyl-phenyl(e)-xanthyliumsalze;

von Frite Dinner.

Diese Verbindungen sind dargestellt worden, um dex Frage nach der Constitution der Fluoresceine näber zu kommen. Sollten nämlich beide Verseifungsproducte, die an Stelle von Methoxyl Hydroxyl enthalten, durch Veslust von Wasser in farbige Verbindungen von unter einander ähnlichen Eigenschaften und gleicher Zusammensetzung sich verwandeln und demnach für beide die gleiche Structur anzunehmen sein, so worde dadurch die Oxoniumbetaïnformel (Brackenformel) für beide bewiesen (und dann auch für Fluoresceïn, Rhodamin u. s. w.), denn ein chinoïder Körper könnte nur aus dem zweiten Isomeren entstehen.

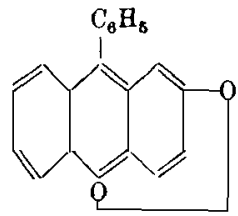

(Brickenformel) einzig mögliche Form des 3-Oxyderivates.

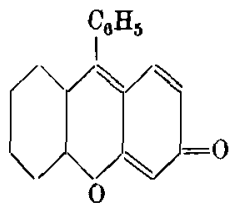

(Chinoide Form) eine der beiden möglichen Formeln des 2-Oxyderivates.

Wir berichten hier über die als Ausgangsproduct bereiteten Methoxylderivate.

Methoxyl(2)-phenyl(9)-xanthylium,

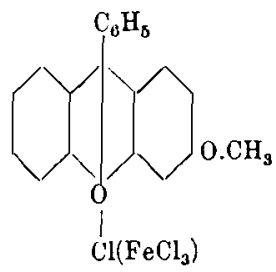

Das Einwirkungsproduct von 2-Methoxylxanthon auf Phenylmagnesiumbromid (im geringen Ueberschusse) in Gegenwart von Benzol und Aether wird mit concentrirter Salzsäure mehr- 
fach ausgezogen. Die saure Lösung ist dunkelroth mit deutlicher Fluorescenz gefärbt. Nach Zusatz von Essigsäure fällt man mit festem Eisenchlorid. Sofort scheiden sich voluminöse, krystallinische, rothe Fällungen des Eisendoppelsalzes aus.

Nach Abpressen krystallisirt man aus einem Gemisch von Eisessig und concentrirter Salzsäure und erbält so schöne, grosse, dunkelrothe Krystalle, die bei 124" schmelzen. Mit Wasser tritt Entfärbung ein unter Bildung der Carbinolbase.

$0,2236 \mathrm{~g}$ gaben $0,4035 \mathrm{CO}_{2}$ und $0,0602 \mathrm{H}_{\mathrm{g}} \mathrm{O}$.

$\begin{array}{ccr} & \text { Berechnet für } & \text { Gefunden } \\ & \mathrm{C}_{30} \mathrm{H}_{15} \mathrm{O}_{2} \mathrm{Cl} . \mathrm{FeCl}_{3} & \\ \mathrm{C} & 49,50 & 49,22 \\ \mathrm{H} & 3,12 & 3,04\end{array}$

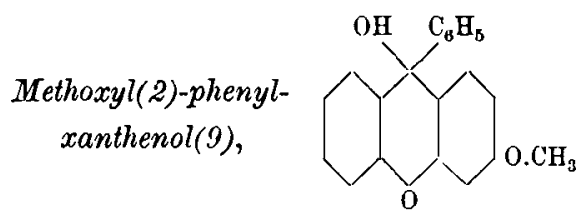

Am einfachsten erhält man diese Carbinolbase durch Verreiben des Oxoniumeisendoppelsalzes mit Wasser bis zur Entfärbung. Man wäscht mit Wasser gut aus, trocknet und hat nun die Base analysenrein. Der Schmelzpunkt liegt bei $133^{\circ}$. In den kohlenstoffhaltigen Lösungsmitteln ist sie leicht löslich. Mit Säuren erhält man die tiefrothen, fluorescirenden Lösungen der Oxoniumsalze, die in zebnprocentiger Salzsäure noch vollkommen beständig sind und erst bei weiterem Verdünnen mit Wasser sich wieder entfärben.

$0,1672 \mathrm{~g}$ gaben $0,4846 \mathrm{CO}_{2}$ und $0,0780 \mathrm{H}_{2} \mathrm{O}$.

$\begin{array}{ccc} & \text { Berechnet fïr } & \text { Gefunden } \\ & \mathrm{C}_{20} \mathrm{H}_{\mathrm{sc}} \mathrm{O}_{3} & \\ \mathrm{C} & 78,91 & 79,09 \\ \mathrm{H} & 5,30 & 5,23\end{array}$

Ueber das entsprechende Xanthen und die Verbindungen, die durch Abspaltung der Methylgruppe entstehen, soll berichtet werden. 


\section{Decker und v. Fellenberg, Ueber Phenopyrylium}

Der Eintritt der Methoxylgruppe verursacht also hier die Vertiefung der Farbe bis zu roth vom orangegelb des einfachen Phenylxanthyliumeisendoppelsalzes.

Methoxyl(3)-phenyl(9)xanthylium,

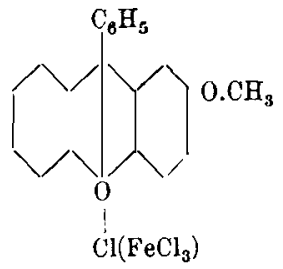

Die Verwandlung des 3-Methoxylxanthons in das Eisendoppelsalz des phenylirten Oxoniumkörpers unterscheidet sich nicht von der soeben beschriebenen Synthese seines Stellungsisomeren.

Das gewonnene Doppelsalz unterscheidet sich von dem eben beschriebenen durch seine viel tiefere Rothfärbung. Es krystallisirt in grossen Nadeln vom Schmelzp. $154^{\circ}$.

$0,1451 \mathrm{~g}$ gaben $0,2620 \mathrm{CO}_{2}$ und $0,0435 \mathrm{H}_{2} \mathrm{O}$.

$\begin{array}{ccc} & \text { Berechnet für } & \text { Gefunden } \\ & \mathrm{C}_{\mathbf{2 0}} \mathrm{H}_{18} \mathrm{Cl} . \mathrm{FeCl}_{3} & \\ \mathrm{C} & 49,50 & 49,25 \\ \text { H } & 3,12 & 3,31\end{array}$

Die mineralsauren Salze sind farbstärker als die des Isomeren und brauchen zur Zersetzung mehr Wasser als diese.

9. Derivate der Phenyl(9)-xanthyliumcarbonsäure(11); von E. Ferrario.

Das Fluoran Iöst sich bekanntlich in Schwefelsăure und zwar mit stark grünlicher Fluorescenz. Im Gegensatze zu den fruberen und den in dieser Abbandlung beschriebenen Phenylxanthyliumsalzen ist dieses schwefelsaure Salz bloss in concentrirter schwefelsaurer Lösung beständig. Auch in concentrirter rauchender Salzsäure ist das Fluoran nicht löslich. Ich komme gleich auf die Ursache dieses auffallend verschiedenen Verhaltens zurück. Die Darstellung der Salze des Fluorans mit 
Säuren lässt sich jedoch unschwer erreichen, wenn man in alkoholischer oder noch besser essigsaurer Lösung operirt. Finige Salze sind auch bereits von Hewitt im festen Zustande dargestellt worden.

Die Ursache der geringen Basicität der Fluoransalze im Vergleiche mit den Phenylxanthyliumsalzen ist kaum in dem Vorhandensein einer sauren 'Carboxylgruppe zu suchen, vielmehr ist sie durçh den Umstand bedingt, dass hier ein dritter Körper auftritt, indem das zuerst entstehende Carbinol mit der Carboxylgruppe sich sofort zum Lactonring - dem Fluoran unter Wasseraustritt schliesst. Während also bei den übrigen Phenylxanthyliumsalzen eine Salzsäure von wenigen Procenten genügt, um das Xanthenol in die Oxoniumsalze zuruckzuverwandeln, ist hier eine Sprengung des Lactonringes nothwendig, und dieser Prozess verlangt eben die Anwendung einer concentrirten Säure. Es ist deshalb verständlich, dass bei einem Gleichgewicht zwischen der Carbinolbase und dem Oxoniumsalze, wie es sich in mässig verdünnter Salzsäure bei nicht carboxylirt:n Oxoniumbasen einstellt, in diesem Falle das ganze Xanthenol und somit auch das ganze Xanthyliumsalz in das in Salzsäure nicht lösliche Fluoran verwandeln wird.

Eisenchloriddoppelsalz des Fluorans,

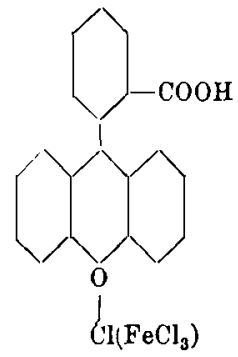

Zum Vergleiche mit den verschiedenen anderen Eisendoppelsalzen haben wir auch das vom Fluoran, durch Zusatz von festes Eisenchlorid enthaltender Salzsäure zur heissen essigsauren Fluoranlösung, erbalten. Es krystallisirt in gelben Nadeln vom Schmelzp. $200^{\circ}$. 
316 Decker und v. Fellenberg, Ueber Phenopyrylium

0,2347 g gaben $0,0375 \mathrm{Fe}_{2} \mathrm{O}_{3}$.

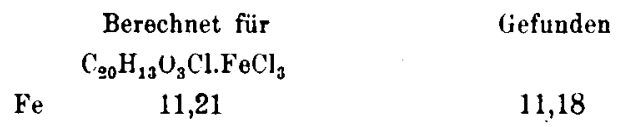

Darstellung ron Dimethylfluoran (Lacton der Dimethylphenylxanthenoncarbonsäure). Zur Darstellung des von Baeyer uod Drews en entdeckten DimethylHuorans benutzten wir folgende Vorscbrift: 20 Theile p-Kresol, i亏 Theile Phtalsäureanhydrid werdeu mit 7 Theilen Zinkchlorid zwei Stunden auf $210^{\circ}$ erwärmt. Die schwarze Schmelze wird nach dem Erkalten in dem doppelten Volumen Alkohol digerirt und abgesogen. Auf dem Saugfilter bleibt das Dimethylfluoran nach Auswaschen mit Alkohol in analysenreinen, weissen Krystallen zurück. Die Ausbeute ist leidlich.

Eisenchloriddoppelsalz. Wie das Fluoransalz wird dieses auch in essigsaurer Lösung bereitet. Es schmilzt bei $215^{0}$ und repräsentirt glänzende, orangegelbe, prismatische Nadeln. Es wird bei $130^{\circ}$ scharf getrocknet.

$$
\begin{array}{ccc} 
& \text { Berechnet für } & \text { Gefunden } \\
& \mathrm{C}_{\mathbf{2} \cdot \mathbf{2}} \mathrm{H}_{17} \mathrm{O}_{\mathbf{3}} \mathrm{Cl} . \mathrm{FeCl}_{\mathbf{3}} & \\
\mathrm{Fe} & 10,61 & 10,63
\end{array}
$$

Beide Fluoransalze sind auch an der feuchten Luft bedeutend weniger beständig als die oben beschriebenen Xanthyliumeisendoppelsalze. 
Kapitel III.

\section{Coeroxonium- und Coerthioniumreihe.}

Zur Darstellung der Verbindungen dieser Reihen wurden die beiden in der Einleitung besprochenen Synthesen benutzt.

\section{Derivate des Coeroxoniums;}

von Enos Ferrario.

Die Synthese aus dem Phenylather des Erythrooxyanthrachinons hat sich unter Einhaltung bestimmter Temperaturgrenzen und Benutzung ron Ruhrwerk zu einer Darstellungsweise ausarbeiten lassen, die über $80 \mathrm{pC}$. der Theorie an Oxoniumsalz liefert. Nichtsdestoweniger ist es im Laboratorium bei weitem bequemer, die Synthese aus Fluoran, die quantitativ verläuft, auszuführen. Die hier beschriebenen Derivate des Coeroxonols-(9) sind theilweise auf dem einen Wege, theilweise auf dem anderen dargestellt worden. Von den Aethern des Coeroxonols ist bis jetzt nur das Aethylderivat beschrieben worden.

Methyläther,

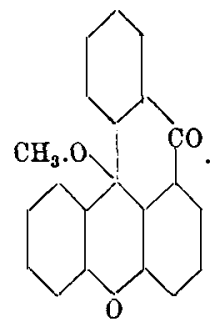

Wird durch Aufkochen von $5 \mathrm{~g}$ des früher beschriebenen Coeroxonols mit $20 \mathrm{ccm}$ absolutem Methylalkohol in farblosen Nadeln vom Schmelzp. $133^{\circ}$ erhalten.

$0,2424 \mathrm{~g}$ gaben $0,7122 \mathrm{CO}_{2}$ und $0,0886 \mathrm{H}_{8} \mathrm{O}$.

Berechnet für $\mathrm{C}_{21} \mathrm{H}_{14} \mathrm{O}_{3}$

C

H

80,23

Gefunden 80,13 


\section{Decker und v. Fellenberg, Ueber Phenopyrylium}

n-Propyläther. Durch Aufkochen des Carbinols mit Normalpropylalkobol enthält man prächtige Krystalle, die bei $151^{\circ}$ schmelzen.

$0,2211 \mathrm{~g}$ gaben $0,6534 \mathrm{CO}_{2}$ und $0,1028 \mathrm{H}_{2} \mathrm{O}$.

$\begin{array}{ccr} & \text { Berechnet für } & \text { Gefunden } \\ & \mathrm{C}_{23} \mathrm{H}_{18} \mathrm{O}_{3} & \\ \mathrm{C} & 80,68 & 80,60 \\ \mathrm{H} & 5,30 & 5,20\end{array}$

Isobutylather. Durch Kochen des Carbinols $(3 \mathrm{~g})$ mit Isobutylalkohol $(6,5 \mathrm{~g})$ (Siedep. 106-108 $)$ entsteht dieser Aether und kommt nach dem Erkalten in weissen Krystallen vom Schmelzp. $132^{\circ}$ heraus. Er ist sebr viel löslicher im Isobutylalkohol als der Aetbylather in dem ihm entsprechenden Alkobol.

$0,2104 \mathrm{~g}$ gaben $0,6226 \mathrm{CO}_{2}$ und $0,1027 \mathrm{H}_{2} \mathrm{O}$.

$\begin{array}{ccr} & \text { Berechnet für } & \text { Gefunden } \\ & \mathrm{C}_{\mathbf{3 4}} \mathrm{H}_{\mathbf{2 0}} \mathrm{O}_{3} & \\ \text { C } & 80,90 & \mathbf{8 0 , 7 0} \\ \text { H } & 5,66 & \mathbf{5 , 6 9}\end{array}$

Verwandlung eines Carbinoläthers in einen anderen durch Aufkochen mit dem entsprechenden Alkohol. Dieser Vorgang, den Decker ${ }^{24}$ ) zuerst bei den Aethern eines Nitro-(5)-brom(3)-chinolanols beobachtet hat, und der dann bei einer grossen Zahl anderer Carbinole nachgewiesen worden ist, lässt sich auch in diesem Falle durchführen. Kocht man z. B. den fruher beschriebenen Aethyläther rom Schmelzp. $148^{\circ}$ mit einer grösseren Menge Metbylalkohol auf, so krystallisirt der Aether die letztereu rom Schmelzp. $133^{\circ}$ aus.

Einwirkungsproduct von Aceton auf Coeroxonol (Acetonylcoeroxon). Eine eigenthumliche Beobachtung haben wir gemacht, als wir versuchten, grössere Mengen der Carbinolbase aus Aceton umzukrystallisiren. Der sonst aus anderen Lösungsmitteln schnell und schön sich ausscheidende Körper hinterblieb als zähflüssiges, dunkelroth gefärbtes Harz, aus dem erst nach

*4) Journ. f. prakt. Chem. 45, 185 (1892). 
wochenlangem Stehen sich Krystalle abschieden. Mit Aether kann der rothe Farbstoff ausgewaschen werden und man erhalt so ein Product, das in Benzol und Chloroform leicht löslich, in Petroläther schwer löslich ist und sich rom Coeroxonol und seinen Aethern bauptsächlich dadurch unterscheidet, dass seine Lösung in kaltem Eisessig sich mit Salzsäure nicht färbt, während durch die Einwirkung von heisser Salzsäure oder Schwefelsäure sich Coeroxoniumsalze zuräckbilden. Die Analyse ergab, dass sich die Carbinolbase mit einem Mol. Aceton unter Austritt von Wasser vereinigt hat, was auf die aldebydische Function des Carbinolhydroxyls zurückzuführen ist. Fosse hat in letzter Zeit ähnliche Beobachtungen gemacht.

$0,1421 \mathrm{~g}$ gaben $0,4228 \mathrm{CO}_{2}$ und $0,0588 \mathrm{H}_{2} \mathrm{O}$.

$\begin{array}{ccc} & \text { Berechnet für } & \text { Gefunden } \\ & \mathrm{C}_{3:} \mathrm{H}_{110} \mathrm{O}_{3} & \\ \mathrm{C} & \mathbf{8 1 , 1 5} & 81,15 \\ \mathrm{H} & 4,74 & 4,63\end{array}$

Der Schmelzpunkt liegt bei $146^{\circ}$.

11. Derivate des Methyl(14)-coeroxoniums (Formel II); ron Leo Stern.

Die nachstehenden Versuche sind in der Absicht unternommen, die von Decker und Ferrario aufgefundene und dann mit Laube ausgearbeitete Synthese von Coeroxoniumsalzen aus dem Phenyläther des Frythrooxyanthrachinons auf ihre weitere Verwendbarkeit zu prüfen.

An Stelle des Phenyläthers wurde der p-hresyläther-(1) genommen. Die Synthese verläuft genau in derselben Weise, nur, wie es scheint, weniger glatt, unter Bildung der Methyl(14)-coeroxoniumsalze (Formel II). 
I.

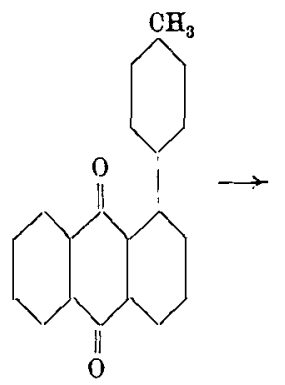

II.

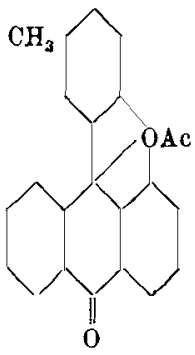

Die Derivate, Carbinol und Carbinoläther und Reductionsproducte zeigen ähnliche Eigenschaften wie die Stammsubstanz. Auch in der Färbung der Oxoniumsalze ist kaum ein Unterschied zu bemerken. Bei dem hohen Molekulargewicht ist eben der Einfluss einer Methylgruppe sebr gering. Haben auch diese Versuche nichts wesentlich Neues in Bezug auf den Reactionsverlauf zu Tage gefördert, so ist doch dadurch ein weiteres wünschenswerthes Beispiel zur Coeroxoniumsynthese geliefort worden.

p-Kresyläther des Erythroxyanthrachinons (Formel I). Diese Verbindung ist nach D. R. P. Nr. 158531 von Friedrich Bayer \& Co. dargestellt worden. Sie ist in den meisten Lösungsmitteln etwas leichter löslich als das niedere Phenolhomologe. Aus Petrolätber erhält man hellgelbe Nädelchen vom Schmelzp. $128,5^{0}$.

$0,1945 \mathrm{~g}$ gaben $0,5732 \mathrm{CO}_{\mathfrak{R}}$ und $0,0829 \mathrm{H}_{\mathrm{g}} \mathrm{O}$.

$\begin{array}{ccc} & \text { Berechnet für } & \text { Gefunden } \\ & \mathrm{C}_{91} \overline{\mathrm{H}}_{14} \mathrm{O}_{\mathrm{B}} & \\ \mathrm{C} & 80,25 & 80,08 \\ \mathrm{H} & \mathbf{4 , 4 8} & 4,70\end{array}$

Eisendoppelsalz des Methyl(14)-coeroxoniums ${ }^{25}$ ) (Formel II). Die Darstellung des Coeroxoniums erfolgt nach der Synthese von Decker und Ferrario durch stundenlanges Erwärmen

25) Betreffend die Numerirung der Coeroxeniumderivate vergleiche diese Annalen 348, 212. 
des soeben beschriebenen Anthrachinonderivates mit $65-70 \mathrm{pC}$. Monohydrat haltender Schwefelsäure. Zweckmässig verwendet man hierbei einen Rübrer. Sobald der grösste Theil des Anthrachinonäthers verschwunden ist, giesst man die dunkelrothe Masse auf Eis und in 15 -procentige Salzsäure, filtrirt und versetzt das noch immer tiefroth gefärbte. Filtrat mit festem Eisenchlorid, bald fällt das Eisendoppelsalz als rothbrauner, krystallinischer Niederschlag aus, der auf der Thonplatte getrocknet bereits sehr rein ist. Aus Eisessig lăsst er sich in rotbbraunen, metallisch, schillernden, mikroskopischen Kryställchen erhalten. Sein Schmelzpunkt liegt bei $232,5-235,5^{0}$. Das Eisensalz zeigt alle charakteristischen Eigenschaften der Coeroxoniumsalze; es verliert beim Zusammenbringen mit Wasser seine Farbe.

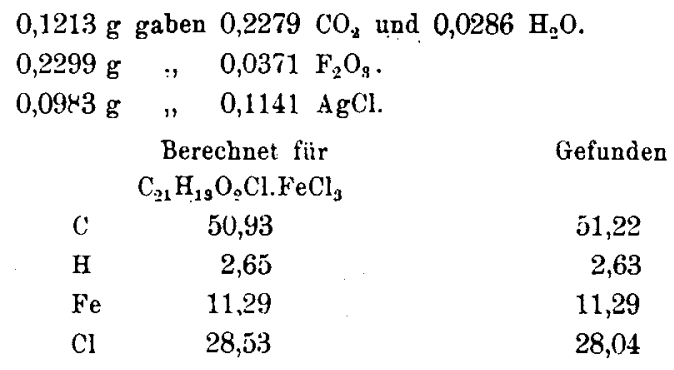

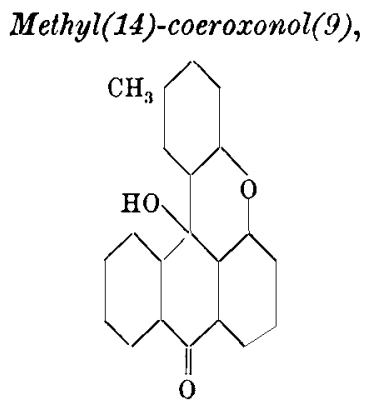

Quantitativ erhält man dieses Carbinol beim Zersetzen irgend einer entsprechenden Oxoniumsalzlösung mit Wasser oder beim Uebergiessen des Eisensalzes mit Wasser. Man siebt dic 
dunkelrothe Farbe schwinden und es hinterbleibt das farblose, krystallinische Carbinol. In den verdunnten Lösungen ist dieser Vorgang so aufaufassen wie alle ihn ähnlichen Fällen, dass sich durch Hydrolyse zuerst das noch rothgefarbte und wasserlösliche Oxoniumbydroxyd bildet; dies ist eine äusserst unbeständige Verbindung und wird sofort in das Carbinol umgelagert. Das rohe Carbinol repräsentirt ein graues, manchmal durch gleichzeitig in geringer Menge entstandene Farbstoffe roth oder violett gefärbtes krystallinisches Product, das am besten aus Benzol unter Zusatz von Petrolather krystallisirt wird, dann bildet es farblose, glănzende Kryställchen vom Schmelzp. $176^{\circ}$. In den kohlenstoff haltigen Lösungen ist der Körper leicht, in Wasser nicht löslich. Mit Săurea giebt er die dunkelrotben Oxoniumsalze zurück.

$0,1235 \mathrm{~g}$ gaben $0,3574 \mathrm{CO}_{2}$ und $0,0538 \mathrm{H}_{2} \mathrm{O}$.

$\begin{array}{ccc} & \text { Berechnet für } & \text { Gefunden } \\ & \mathrm{C}_{21} \mathrm{H}_{10} \mathrm{O}_{3} & \\ \mathrm{C} & 80,23 & 79,99 \\ \mathrm{H} & \mathbf{4 , 4 8} & \mathbf{4 , 4 9}\end{array}$

Aethyläther. Krystallisirt man die Carbiuolbase aus kochendem Aetbylalkohol, so erbält man Nadeln dieses bei $139^{\circ}$ schmelzenden Körpers.

$0,1266 \mathrm{~g}$ gaben $0,3715 \mathrm{CO}_{\mathrm{z}}$ und $0,0611 \mathrm{H}_{\mathrm{z}} \mathrm{O}$.

Berechnet für

$$
\mathrm{C}_{29} \mathrm{H}_{18} \mathrm{O}_{3}
$$

c

H
80,67

5,20 (iefunden

80,10

5,39 
12. Derivate des Dimethyl(4,14)-coeroxoniums (Formel II); von Enos Ferrario.
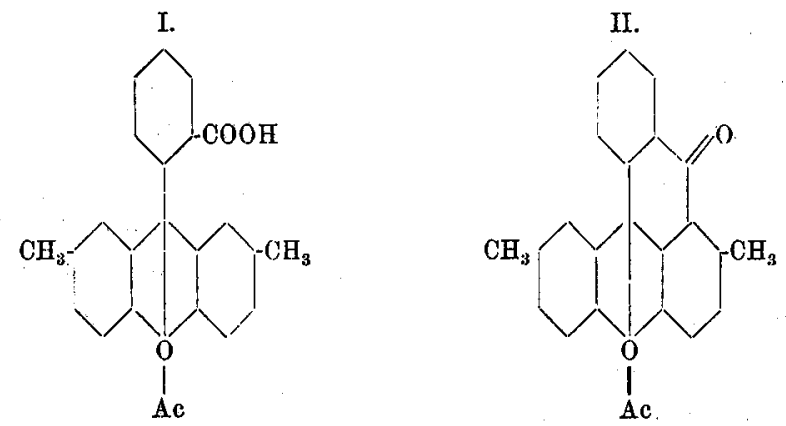

Das leicht zugängliche Dimethylfluoran ist das Ausgangsproduct zu einer Coeroxoniumsynthese, wie sie obenstehende Formeln illustriren, benutzt worden.

Dimethyl(4,14)-coeroxonol-(9) (Carbinolbase der Oxoniumsalze),<smiles>CC1CCC2OC3CC[C@@H](C)CC3C2C(O)(C2CCCCC2O)C1</smiles>

Diese Carbinolbase ist früher nur kurz erwähnt ${ }^{26}$ ) und irrthümlich als 3,13 statt 4,14 bezeichnet worden. Zu ihrer Bereitung ist zu bemerken, dass die Condensation des Dimethylfluorans von Baeyer \& Drewsen am besten mit einer Schwefelsäure von $20 \mathrm{pC}$. Anhydridgehalt ausgeführt wird. Man wartet einige Minuten, bis sich eine Glasstabprobe vollkommen in zehnprocentiger Schwefelsäure löst. Die Farbe der nun Dimethylcoeroxoniumsalz enthaltenden Schwefelsäureschmelze ist

${ }^{26}$ ) Diese Annalen 348, 224. 
dann ein reines Kirschroth ohne Fluorescenz ${ }^{27}$ ). Die Fluorescenz rührt von dem Oxoniumsulfat des Dimethylfluorans her. Operirt man unter Einbaltung dieser Bedingung und unter Eiskühlung, so erhält man nach dem Eingiessen in englische Schwefelsäure, Verdünnung mit Wasser und Abstumpfen der Säure mit Ammoniak unter guter Küblung einen grtunlichen Niederschlag, der nach mehrfacher Krystallisation aus Benzol den Schmelzp. $170^{0}$ (früher $152^{\circ}$ angegeben) zeigt. Das Carbinol ist in den koblenstoffhaltigen Lösungsmitteln leicht, in Wasser nicht löslich. Anilin nimmt es in der Wärme mit blauer Farbe auf, Mineralsäuren lösen es mit prächtiger, rother Farbe, die beim Verdünnen der Säuren unter $5 \mathrm{pC}$. allmählich verblasst. Es entsteht eine Trübung, und die benzollöslicbe, farblose Carbinolbase fällt aus, die sich aus Benzol krystallisiren lässt.

$0,2019 \mathrm{~g}$ gaben $0,5979 \mathrm{CO}_{\underline{a}}$ und $0,0866 \mathbf{H}_{\mathbf{g}} \mathrm{O}$.

$\begin{array}{ccc} & \text { Berechnet für } & \text { Getunden } \\ & \mathrm{C}_{\mathrm{gs}} \mathrm{H}_{16} \mathrm{O}_{3} & \\ \mathrm{C} & 80,46 & 80,74 \\ \mathrm{H} & 4.91 & \mathbf{4 , 7 9}\end{array}$

.Tethyläther des Dimethyl(4,14)-coeroxonols(9), $\mathrm{C}_{89} \mathrm{H}_{18} \mathrm{O}_{9}$. Durch Aufkochen der Carbinolbase mit absolutem Methylalkohol erhält man den in kleinen, farblosen Krystallen herauskommenden Methyläther vom Schmelzp. $10 \mathrm{a}^{0}$.

Aethyläther. Denselben erbält man durch Krystallisation des Carbinols aus Aethylalkohol; kleine Krystalle vom Schmelzpunkt $145^{\circ}$.

$0,2130 \mathrm{~g}$ gaben $0,6260 \mathrm{CO}_{2}$ und $0,1077 \mathrm{H}_{\mathrm{y}} \mathrm{O}$.

$\begin{array}{ccc} & \text { Berechnet für } & \text { Gefunden } \\ & \mathrm{C}_{24} \mathrm{H}_{20} \mathrm{O}_{\mathbf{s}} & \\ \mathrm{C} & 80,88 & 80,80 \\ \mathrm{H} & \mathbf{5}, 65 & 5,70\end{array}$

27) Die Fluorescenz rührt von dem Oxoniumsulfat des Dimethylfluorans her. 
Die Salze des Dimethylcoeroxoniums (Formel II). Das Carbinol zeigt mit Mineralsäuren eine prächtige himbeerrothe Färbung und ist verbältnissmässig basisch; mit zehnprocentiger Salzsäure erhält man leicht Salzlösungen. Das Sulfat ist in concentrirter Schwefelsäure schwer löslich und fällt in dunkelrothen Krystallen aus. Das aus dem reinen Producte gewonnene Eisendoppelsalz zeigt eive purpurrothe Farbe und schmilzt bei $210^{\circ}$. Die Verbrennung ist früher mitgetheilt worden.

$0,3003 \mathrm{~g}$ gaben $0,0449 \mathrm{Fe}_{2} \mathrm{O}_{3}$.

$$
\begin{aligned}
& \text { Berechnet fiir Gefunden } \\
& \mathrm{C}_{92} \mathrm{H}_{13} \mathrm{O}_{2} \mathrm{Cl}_{\text {. FeCl }} \\
& \mathrm{Fe} \quad 10,77 \quad 10,36
\end{aligned}
$$

Dimethyl(4,14) - coeroxenol(10) - acetat. Reducirt man die Carbinolbase oder das entsprechende Oxoniumsalz, so erhält man das stark grun fluorescirende, mit orangerother Farbe in Natronlauge lösliche Coeroxenol (10), das aber in Folge seiner grossen Oxydirbarkeit nicht leicht zu isoliren ist. Viel leichter ist das Acetat zu erbalten, das man durch Reduction in Essigsäureanhydridlösung vermittelst Zinkstaub erbält. Dieser Körper hat eine gelbe Farbe; er schmilzt, aus Essigsäure krystallisirt, bei $230^{\circ}$. Die Lösung ist gelb mit schön grüner Fluorescenz. In Natronlauge ist der Körper nur durch Kochen unter Verseifung als Coeroxenol in Lösung zu bringen. Es sieht, wie alle die bier beschriebenen Verbindungen, seinen niederen Homologen täuschend ähnlicb.

13. Derivate des Methyl(14)-coerthioniums (Formel II); von August Würsch.

In derselben Weise wie Decker und Warsch vom Thiophenoläther des Anthrachinons zum Coerthionium gelangt sind, wurde die Synthese mit dem homologen p-Thiokresoläther ausgeführt. 
I.

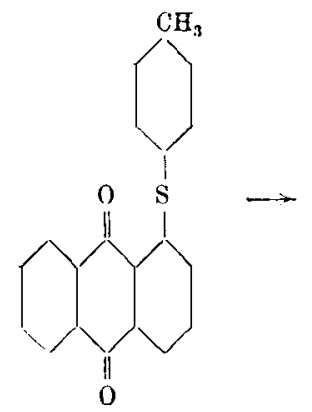

II.

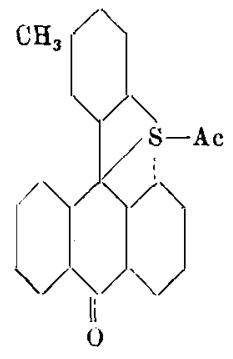

Darstellung von p-Thiokresol, $\mathrm{CH}_{3} \mathrm{C}_{6} \mathrm{H}_{4} \mathrm{SH}$. Das Arbeiten mit Thiophenol ist im Laboratorium wegen seines unangenebmen und durchdringenden Geruches nicht angenehm. Seine Verwendung im Grossen ist aus demselben Grunde unmöglich. Es ist deshalb interessant $\mathbf{z u}$ constatiren, dass das homologe p-Thiokresol, das zu vielen Zwecken als Ersatz dienen kann, diese unangenehmen Eigenschaften nicht besitzt. Im Laboratorium kann es offen tagelang stehen, ohne dass sich jemand über Belästigung beklagt. Als fester Körper ist es auch handlicher als sein flüssiges Homologe. Es ist auch bei weitem zugänglicher als das Thiophenol, da es in beliebiger Menge aus dem werthlosen Nebenproducte der Saccharinfabrikation, dem p-Toluolsulfonsäurechlorid, dargestellt werden kann.

An Stelle von Zinn und Salzsäure haben wir Zink oder Eisen und Salzsäure angewandt. Wir benutzten gewöhnlich auf 20 Theile Sulfonsäurechlorid $74 \mathrm{~g}$ Eisenfeile, $30 \mathrm{~g}$ Essigsäure und fagten allmählich zu der auf dem Wasserbade siedenden Masse noch $200 \mathrm{ccm}$ 15-procentige Salzsäure zu. Nach fünf Stunden langem Digeriren wurde das Thiokresol im Dampfstrome abgetricben und durch Filtration isolirt. So erhält man ein reines Product in einer Ausbeute von etwa 50 pC. der Theorie. Wir begnugten uns damit bei der grossen Billigkeit des Ausgangsproductes, glauben aber, dass durch Abănderung der Verhältnisse eine höhere Ausbeute erzielt werden kann. 
p-Kresyläther des Thio(1)-anthrachinons (Formel I), $\mathrm{C}_{14} \mathrm{O}_{2} \mathrm{H}_{7} . \mathrm{S}_{6} \mathrm{C}_{6} \mathrm{H}_{4} \mathrm{CH}_{3} .8 \mathrm{~g}$ Kaliumbydroxyd und $10 \mathrm{~g}$ Thiokresol löst man in absolutem Alkobol; nach Abtreiben des Alkohols erhitzt man das Kaliunsalz noch auf $150^{\circ}$ und zerreibt es in einem Mörser innigst mit $24 \mathrm{~g} \alpha$-antbrachinonsulfonsaurem Kalium. Die Masse wird nun zwei Stunden auf $180^{\circ}$ erwärmt, dann mit Wasser ausgekocht. Der unlösliche Ruckstand ist der Thioätber. Ausbeute $20 \mathrm{~g}$. (Nach dem Patent von Fr. Bayer \& Co. dargestellt.)

Besser geht die Darstellung mit Nitroanthrachinon (10 g), das mit Kaliumbydrat $(5,6 \mathrm{~g})$, Thiokresol $(5,6 \mathrm{~g})$ und $50 \mathrm{~g}$ Alkohol auf dem Wasserbade zwei Stunden erhitzt wird. Ausbeute $75 \mu \mathrm{C}$. der Theorie. Aus Eisessig oder Benzol erbält man schöne, orangerotbe Nadeln, die bei $223-225^{\circ}$ schmelzen.

$0,1420 \mathrm{~g}$ gaben $0,3965 \mathrm{CO}_{2}$ und $0,0520 \mathrm{H}_{8} \mathrm{O}$.

$\begin{array}{ccr} & \text { Berechnet für } & \text { Gefunden } \\ & \mathrm{C}_{\mathbf{2 1}} \mathrm{H}_{14} \mathrm{SO}_{1} & \\ \mathrm{C} & 76,37 & 75,68 \\ \mathrm{H} & 4,34 & 4,16\end{array}$

Methyl(14)-coerthioniumsalze (Formel II). $13 \mathrm{~g}$ des Thioanthrachinonäthers werden fein zerrieben und unter Rubren in $200 \mathrm{~g} 85 \mathrm{pC}$. Monohydrat enthaltende Schwefelsäure auf $180^{\circ}$ erhitzt. Der Aether löst sich grün und nach etwa 24 Stunden nimmt die Schmelze die violette dichroitische Farbe der Coerthioniumsalze an. Man lässt erkalten, filtrirt durch Glaswolle, wobei der unveränderte Aether auf dem Filter bleibt. Es empfieblt sich, Borsäure zuzugeben, welche die Sulfonsăurebildung zurückzudrängen scheint. Die Thioniumsalzlösung giesst man in $600 \mathrm{ccm} 1 j$-procentige Salzsăure.

Die Lösungen des Thioniumsalzes sind in grosser Verdünnung rein grün, in dicker Schicht sehen sie kupferroth aus. Fluorescenz kommt ihnen nicht zu. Man fält mit Eisenchlorid das schwarzgrüne Eisendoppelsal $z$ aus und krystallisirt es aus Eisessig um. Sein Schmelzpunkt liegt bei $240^{\circ}$. Mit 
Wasser wird das Salz hellgrün und dann farblos unter Bildung von Carbinolbase.

$0,2506 \mathrm{~g}$ gaben $0,4484 \mathrm{CO}_{2}$ und $0,0606 \mathrm{H}_{\mathrm{a}} \mathrm{O}$.

$\begin{array}{ccr} & \text { Berechnet für } & \text { Gefunden } \\ & \mathrm{C}_{\mathbf{2 1}} \mathrm{H}_{2 \mathrm{a}} \mathrm{OSFeCl}_{\star} & \\ \mathrm{C} & 49,30 & 48,80 \\ \text { H } & 2,62 & \mathbf{2 , 6 8}\end{array}$

Methyl(14)-coerthionol(9),

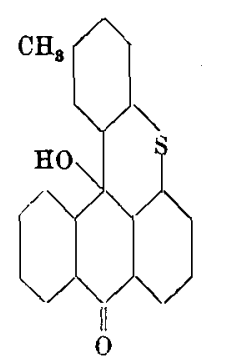

Die rohe Fällung der Carbinolbase, wie man sie aus den Oxoniumsalzen mit Wasser und Alkalien erbält, die noch dunkel gefärbt ist, wird wiederholt in Salzsäure gelöst und mit Wasser wieder ausgefällt. Man trocknet schliesslich den Niederschlag recht gut im Exsiccator und krystallisirt aus Benzol um. Er stellt ein fast weisses, krystallinisches Pulver vom Schmelzpunkt $235^{0}$ dar.

$0,2044 \mathrm{~g}$ gaben $0,5349 \mathrm{CO}_{2}$ und $0,0783 \mathrm{H}_{2} \mathrm{O}$.

$\begin{array}{cc}\text { Berechnet für } & \text { Gefunden } \\ \mathrm{C}_{21} \mathrm{H}_{14} \mathrm{O}_{2 .} \mathrm{S} & \\ 76,36 & 76,30 \\ \mathbf{4 , 2 4} & 4,27\end{array}$

Die Lösungen des Coerthioniumsalzes werden durch alle sauren und basischen Reductionsmittel in das Methyl(14)coerthiënol(10) $\left(\mathrm{C}_{21} \mathrm{H}_{14} \mathrm{SO}\right)$ umgewandelt, einen gelben Körper, der von den kohlenstoffhaltigen Lösungsmitteln mit gelber Farbe und stark grünlicher Fluorescenz aufgenommen wird. In Wasser unlöslich, geht er in Alkalien als blaugrünes Phenolsalz in Lösung. In saurer Lösuug oxydirt giebt er die Thioniumsalze zurück. Sein Schmelzpunkt liegt bei $247^{\circ}$. 
Kapitel IV.

\section{Coerbioxylium and Isologe.}

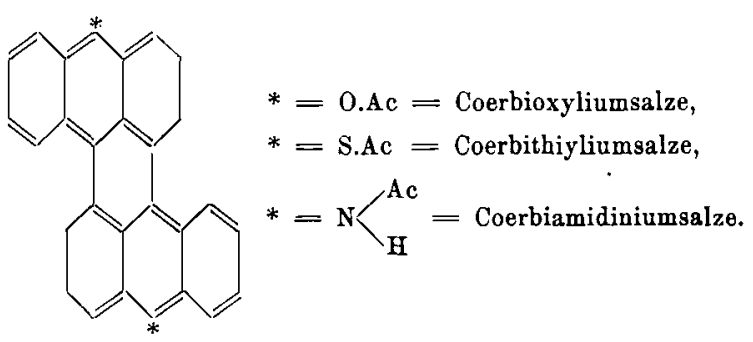

Diese gleichzeitig zwei hetherocyklischen Ringe enthaltenden Körper unterscheiden sich von dem Coeroxonium u. s. w. durch den Ersatz der Carbonylgruppe durch eineu zweiten ,angeschweissten" Phenopyryliumring ${ }^{28}$ ).

Das Stickstoffisologe der unter der Formel (III) angeführten Körper ist zuerst von den Farbenfabriken Bayer \& Co. gewonnen worden. D.R.P. 126444 . Wir nennen es hier unter gleicher Ableitung Coerbiamidin.

28) Für die Namen ist die Endung „ylium" statt "onium" genommen worden, da sie im letzten Falle das Vorhandensein einer Carbonylgruppe andentet. Es mag jedoch nicht unerwähnt bleiben, dass man sich diese complexen Ringe durch Verschweissen von zwei Xanthylium- oder Thioxanthyliumringen in die Stellung 9 und 4 unter Austritt von vier Wasserstoffatomen entstanden denken kann. Eine derartige Ableitung ist zu dem Verständniss ihrer Eigenart von Belang. Bei dieser Formulirung kommt jedoch nicht zur Geltung, dass das Reductionsproduct, das "Coerbioxen" u.s. w., ein richtiges Anthracenderivat ist [Diphenyl $(9,10)$-anthracenbioxyd], und würde die Ableitung des letzteren vom Xanthen durch Austreten von sechs Wasserstoffatomen gesucht erscheinen. 


\section{Coerbioxyliumderivate;} von Enos Ferrario.

I.

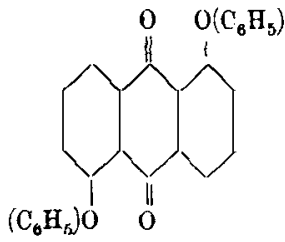

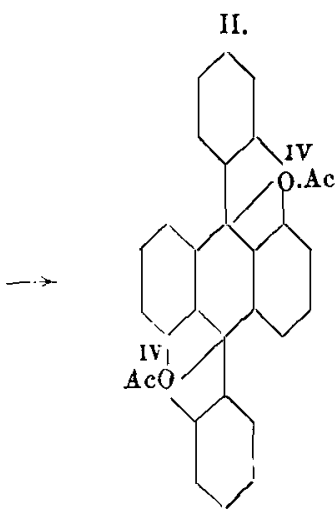

Weniger befriedigende Resultate als bei der Condensation des Phenyiathers des Erythrooxyanthrachinons hatten wir aufzuweisen, als wir einen Dipbenyläther des Anthrarufins (I) durch innere Ringschliessung zu einem Salz condensiren wollten. Auch bei Anwendung von nur $65-70 \mathrm{pC}$. Monohydrat haltender Schwefelsăure und von Temperaturen, die noch nicht $150^{\circ}$ ubersteigen, ist der grösste Theil des Diăthers sehr bald verschwunden, wahrend sich nur eine geringe Menge eines in Form von Eiseudoppelsalz fällbaren Oxoniumsalzes (II) gebildet hat. Der Freundlichkeit der Direction der Farbfabriken Friedrich Bayer \& $\mathrm{Co}$, verdanken wir eine grössere Menge des Diäthers und konnten in Folge dessen trotz der ungunstigen Ausbeute die Reaction studiren. Unter Anwendung von Phosphorsăure allein oder zusammen mit Schwefelsaure lăsst sich das Condensationsproduct einheitlich fassen.

Die Lösungen der Coerbioxoniumsalze in Sâuren sind rein grün. Es durchläuft also die Scbmelze wăhrend des Condensationsprozesses alle olivenfarbigen und grünen Nüancen, die zwischen der rothen Lösungsfarbe des Anthrarufinäthers in Schwefelsäure und rein grin liegen. Ist letztere Farbe er- 
reicht, so giesst man die Scbmelze in die 10 - bis 20 -fache Menge 15-procentiger Salzsüure, womöglich in der Wärme (da sonst das schwer lösliche Sulfat sich ausscheiden würde), filtrirt von dem etwa ausfallenden unveränderten Anthrarufinäther ab und fallt mit festem Eisenchlorid. Die Flüssigkeit färbt sich sofort schwarz, und es fält ein krystallinischer, schwarzer Niederschlag aus, der abgesogen und aus salzsäurehaltigem Eisessig umkrystallisirt werden kann. Es ist dieses das Eisendoppelsalz des Coerbioxoniums ${ }^{2 ! !}$ ) (Formel II'.

$0,1609 \mathrm{gr}$ gaben $0,245 \mathrm{j} \mathrm{CO}$, und $0,0034 \mathrm{H}, \mathrm{O}$.

$0,17: 31$ ir $\quad, \quad 0,1996 \mathrm{AgCl}$.

$0,3140 \mathrm{~g} \quad, \quad 0,0676 \mathrm{Fe}_{0} \mathrm{O}_{3}$.

Berechnet fïr

cietunden $\left(1, \mathrm{H}_{14} \mathrm{O}_{2}, \mathrm{CI}_{8} \mathrm{Fe}_{2}\right.$

(.) $\quad 41,38$

41,69

HI $\quad 1,87$

$2,4.2$

fe $\quad 14,80$

14,94

(1) 28,02

28,07

Die Salze der Bioxoniumbase sind dunkelgrün. Das Sulfut ist ein schwarzgrunes, krystallinisches Pulver; das Hexabromid ein rothbrauner Nicderschlag; das Jodid ein schwarzer Niederschlag, der sehr leicht in das Coerbioxen übergeht. Beim Verdünnen mit Wasser entsteht in den grünen Lösungen zuerst eine vorübergehende röthliche Färburg, die vielleieht von der halb umgelagerten Verbindung, die gleichzeitig Carbinolbase als Oxoniumsalz ist, herruhrt.

Wir halten es nicht für unwahrscheinlich, dass die Con-

29) Durch diese Analysen ist die Thatsache der doppelten Ringschliessung festrelegt; das mögliche Zwischenproduct mit nur einmal geschlossenem Ringe, das i-Phenoxylcoeroxonium, haben wir nicht fassen könuen: seine Carbinolbase wïrde sowohl dem P'henyläther, als auch der Bioxenolbase isomer sein, sein Fisensalz aber bedeutend abweichende Zahlen zeigen. Vielleicht bringt die Aufklïrung über das Verbleiben des griossten Theils des Anthra. rutinithers während der ('ondensation auch eine Aufklärung iber diestell l'unkt. 
densation eine vorhergehende Reduction der Carbonylgruppe verlangt.

Coerbioxendiol (Carbinolbase des Coerbioxoniums).

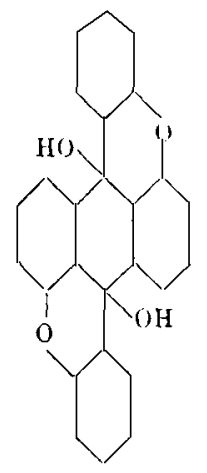

Behandelt man das Fisensalz mit Wasser oder irgend eine grüne Coerbioxoniumlösung mit viel Wasser oder Alkalien, so erhält man ciu violettes, krystallinisches Pulver, das sich aus den verschiedenen Lösungsmitteln umlirystallisiren lässt.

$0,1907 \mathrm{~g}$ gaben $0,5550 \mathrm{CO}_{2}$ und $0,0724 \mathrm{H}_{2} \mathrm{O}$.

$\begin{array}{ccc} & \text { Berechnet für } & \text { Ciefinden } \\ & \mathrm{C}_{26} \mathrm{H}_{10} \mathrm{O}_{4} & \\ \mathrm{C} & 79,56 & 79,42 \\ \mathrm{H} & 4,11 & 4,24\end{array}$

Es treten dabei prachtvolle Farberscheinungen zu Tage. Je nach der Natur des Lösungsmittels variirt die Farbe der Flüssigkeit von violettblau bis violettroth mit einer intensiven, sehr charakteristischen, kupferbraunrotben Fluorescenz, die den Lösungen bei einer Verdünung, wo die Farbe bereits nicht mehr zu bemerken ist, noch anhaftet.

Möglicherweise rühren die Farberscheinungen in der $L_{\alpha}$ sung der Carbinolbase von stets auftretenden Zersetzungsproducten her, und es ist nicht ausgeschlossen, ja wahrscheiulich, dass dieser Körper in vollkommen reinem Zustande farblos ist. 
und hohere homologe und isologe Pyryliumringe. 333

Coerbioxen, $\mathrm{C}_{26} \mathrm{H}_{14} \mathrm{O}_{2}$ (Leukokörper des Coerbioxyliums),

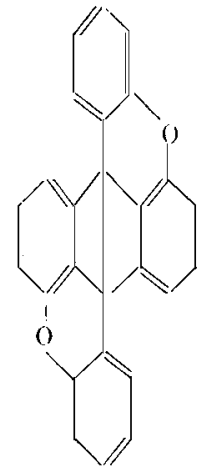

Reducirt ${ }^{30}$ ) man irgend eine grüne Coerbioxyliumlösung in Säure, so fällt ein orangerother Körper aus, der in Aether, Benzol u. s. w. mit orangerother Farbe und intensiver grüner Fluorescenz löslich ist.

Schüttelt man eine derartige Lösung mit oxydirenden Säuren: Salpetersäure, arsensäurehaltiger Schwefelsäure u. s. w., so bildet sich momentan grünes Coeroxoniumsalz zurück, während das kohlenstofthaltige Lösungsmittel farblos wird. Dieses durch Oxydation und Reduction bedingte Farbenspiel kann beliebig oft wiederholt werden und hat seinen Grund in den conjugirten Doppelbindungen der beiden entstehenden Körper ${ }^{31}$ ), die eine Lmlagerung ausserordentlich leicht machen.

Zur Beurtheilung der Constitution des Coerbioxens ist der Umstand massgebend, dass ihm die Fähigkeit des Sublimirens zukommt. Das krystallinische, rothe Sublimat, das sich aus den farbigen Dämpfen condensirt, zeigt alle Reactionen des gefällten Körpers. Diese hohe Beständigkeit steht im besten Ein-

30) Ein alkalilösliches, dem Coeroxenol-(10) aualoges Product entsteht nicht, da hier keine Carbonylgruppe entstanden ist.

3) Wir haben es hier mit einer ,umgekehrten Kiipe" zn thun, insofern als der unlïsliche körper sich nicht durch Oxydation, sondern durch Reduction ausscheidet und der Prozess nicht in alkalischer Lösung, sondern in saurer verläuft. 
klange mit dem einfachen Bau der Verbindung, die dem früher beschriebenen Coeroxen an die Seite gestellt werden kann. Wie dieses sich rom Phenyl-(9-)anthracen ableitet, leitet sich jenes rom Ibiphenyl-(9,10-)anthracen $a b$; allen vier Verbindungen kommt die Fluoreseenz zu.

\section{Coerbithiyliumderivate;}

ron August Würsch.

Diphenylüther des Dithio-(1,5-)anthrachinons (Formel I). Dieser Aether ist bereits in der Patentschrift von Friedrich Bayer \& Co. beschricben. Danach haben wir $10 \mathrm{~g}$ Kaliumhydroxyd, 12 g Thiophenol, $15 \mathrm{~g}$ Dinitroantbrachinon-(1,5) (in fein vertheilten Zustande) in alkoholischer Lösung während zwei Stunden in Sieden erbalten. Zuerst färbt sich das Gemisch grünlich, schliesslich wird es braun. Die I'msetzung erfolgt quantitativ und man bekommt fast die theoretische Ausbeute des Aethers, der sich krystallinisch ausscheidet. Zum Umkrystallisiren des schwer lüslichen Aethers ist Xylol den anderen Lösungsmitteln vorzuzichen. Orangerothe Tafeln rom Schmelzp. $247^{\circ}$.

$0,2098 \mathrm{~g}$ gaben $0,5680\left(\mathrm{CO}_{2}\right.$ und 0,0720$) \mathrm{HI}_{2} \mathrm{O}$.

$\begin{array}{cc}\text { Berechnet fiir } & \text { ciefinden } \\ { }^{1}, \mathrm{H}_{1:} \mathrm{O}_{2} \mathrm{~s}_{2} & \\ 73,05 & 73,29 \\ 3,77 & 3,81\end{array}$


und höhere homologe und isologe Pyryliumringe. 335

Condensation zu Coerbithioniumsalzen (Formel II), I.
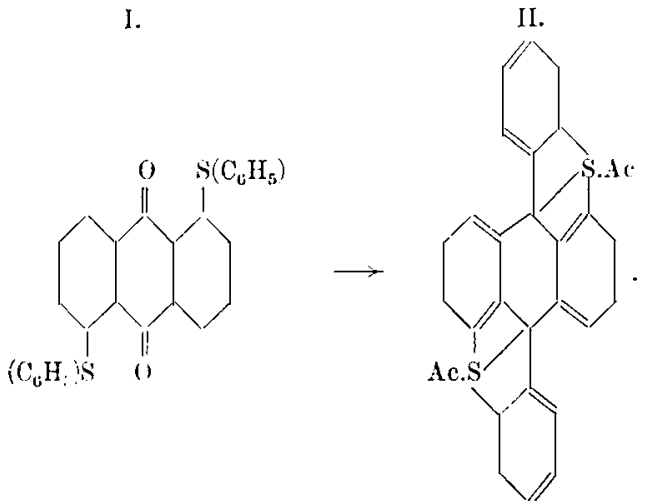

Die bei der Coerthioniumsynthese angegebenen Vorschriften bewähren sich in diesem Falle nicht. Man ist entweder genöthigt, mit $90 \mathrm{pC}$. Monohydrat haltender Schwefelsäure mehrere Stunden auf $200^{\circ} \mathrm{zu}$ erhitzen, wobei sich jedoch erhebliche Mengen Sulfonsäure bilden, oder mit Phosphorsäure zu arbeiten, die reine Producte liefert, aber mehrere Tage lang dauerndes Erwärmen auf über $200^{\circ}$ erfordert. Man giesst die möglichst reingrüne Schmelze, nachdem sie von Kohletheilchen und unverändertem Diäther durch Filtriren über Glaswolle befreit worden ist, in die zehnfache Menge 15-procentiger Salzsäure aus und fällt mit Eisenchlorid, oder man isolirt das schwer lösliche, dnnkelgrune, krystallinische Sulfat durch Verdunnen der Schmelze mit dem zweifachen Volumen Wasser. Die mit Salzsäure und Eisenchlorid in der Wärme versetzte, dunkelgrüne Lösung eines Coerbithioniumsalzes liefert ein schwarzes, krystallinisches, sebr schwer löslicbes, allmählich ausfallendes Eisendoppelsalz, das mit 15-procentiger Salzsäure vom Eisenchlorid reingewaschen und bei $130^{\circ}$ getrocknet wird.

$0,1715 \mathrm{~g}$ gaben $0,2465 \mathrm{CO}_{3}$ und $0,0270 \mathrm{H}_{2} \mathrm{O}$.

$\begin{array}{ccc}0,1713 \mathrm{~g} & \text { \#, } 0,2463 \mathrm{AgCl} & \\ & \text { Berechnet für } & \text { Gefunden } \\ & \mathrm{C}_{\mathbf{9} 6} \mathrm{H}_{4} \mathrm{~s}_{2} \mathrm{Cl}_{3} \mathrm{Fe}_{2} & \\ \mathrm{C} & 39,48 & 39,58 \\ \mathrm{H} & 1,77 & 1,78 \\ \mathrm{Cl} & 35,95 & 35,54\end{array}$


Die schwarze, krystallinische Substanz zeigt beim Zerreiben einen grünen Stich und schmilzt bei $258-260^{\circ}$. Das Hexabromid ist ein olivenfarbiger Niederschlag.

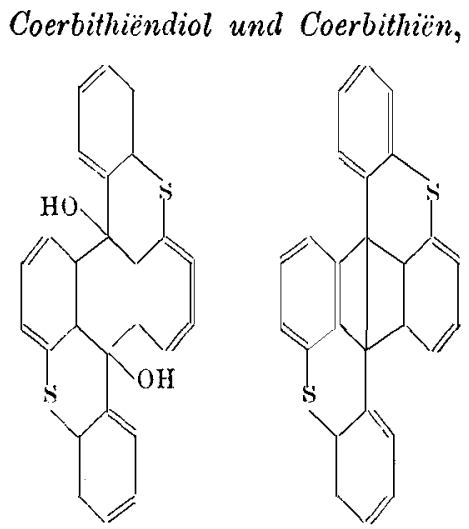

Die mit Phosphorsäure dargestellten grünen Cocrbithioniumsalze gehen bei reichlichem Zusatz von Wasser allmählich in einen farblosen Körper uber, den man aus Eisessig krystallisiren kann und der den Schmelzp. $248^{\circ}$ besitzt. Es ist jedoch beim Ausfällen und Umkrystallisiren jedes überfüssige Erwärmen zu vermeiden, sonst stellen sich äusserst intensive Färbungen und Fluorescenzerscheinungen ein. Dieses Verbalten bestätigt auch die Vermuthung, dass das oben beschriebene isologe Coerbioxendiol ebenfalls ein farbloser Körper ist und die auftretenden Farberscheinungen von Nebepproducten herrühren. Reducirt man die Salze in saurer Lösung, so fällt sofort ein orangerother Körper aus, der in verdünten Säuren, Wasser und Alkalien unlöslich ist, von den koblenstoffbaltigen Lösungsmitteln aber leicht unter intensiver, gelbrother Fluorescenz aufgenommen wird. Oxydirende Säuren schutteln aus diesen letzteren Lösungen wieder die grünlich fluorescirenden Coerbitbioniumsalze aus. Das Coerbitbiën lässt sich unzersetzt sublimiren. Für diese Erscheinung bat der Eine von uns eine Erklärung in der Theorie der conjugirten Oniumsysteme gegeben. 
Di-p-kresyläther des Dithio-(1,j-)anthrachinons,

I.

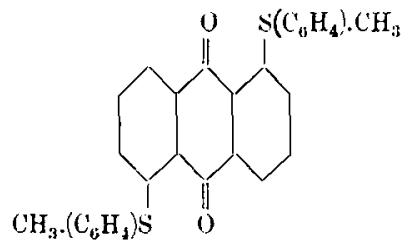

II.

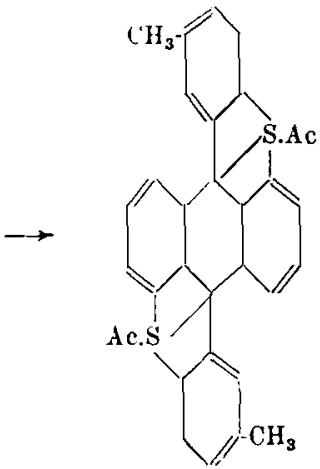

$10 \mathrm{~g}$ Thiokresol, $8 \mathrm{~g}$ Kaliumhydroxyd und $12 \mathrm{~g}$ Dinitroanthrachinon- $(1,5)$ werden in circa $175 \mathrm{ccm}$ Alkobol zwei Stunden lang gekocht. Das Gemisch wird zuerst gelb, dann grün, während der Diäther sich als krystallinisch wohl unterscheidbares, rothbraunes Pulver ausscheidet. Man saugt ab, trocknet und krystallisirt aus Xylol; prächtige, rothbrauie Krystalle vom Schmelzp. $249^{\circ}$.

$0,2120 \mathrm{~g}$ gaben $0,5775 \mathrm{CO}_{2}$ und $0,0837 \mathrm{H}_{2} \mathrm{O}$.

$\begin{array}{ccc} & \text { Berechnet für } & \text { Gefinden } \\ & \mathrm{C}_{2 \times} \mathrm{H}_{20} \mathrm{~S}_{2} \mathrm{O}_{2} & \\ \mathrm{C} & 74,36 & 74,31 \\ \mathrm{H} & 4,20 & 4,40\end{array}$

Dimethylcoerbithiyliumsalze (Formel II). Die intramolekulare Wasserabspaltung aus dem soeben beschriebenen Anthrachinonderivat geht nicht glatt mit Schwefelsäure der verschiedensten Concentrationen. Wir haben schliesslich 90 Stunden mit einem Gemisch concentrirter Schwefelsäure und Phosphorsäure auf $200^{\circ}$ erhitzen müssen. Die erkaltete Schmelze wird, sobald sie rein grüne Farbe besitzt, in eine Salzsäure von $15 \mathrm{pC}$. Gebalt, die man vorher auf $50^{\circ}$ erwärmt hat, eingegossen, nach einiger Zeit wieder filtrirt und mit Eisenchlorid gefăllt. Nach dem Abkühlen erhält man glänzende, schwarze Krystalle: 
des Eisendoppelsalzes von Dimethylbithiylium, die sich aus Eisessig umkrystallisiren lassen und bei $204^{\circ}$ schmelzen.

$0,1580 \mathrm{~g}$ gaben $0,2812()_{0}$ und $\left.0,0378 \mathrm{H}_{2} \mathrm{O}\right)$.

$\begin{array}{ccc} & \text { Berechnet fiir } & \text { (iefunden } \\ & C_{2 \rightarrow} \mathrm{H}_{18} \mathrm{~S}_{z} \mathrm{Cl}_{8} \mathrm{Fe}, & \\ \mathrm{C} & 41,28 & 41,48 \\ \mathrm{H} & 2,21 & 2,28\end{array}$

Mit Wasser tritt Eutfärbung des Salzes ein, denn das Dimethylcoerbithiëndiol, $\mathrm{C}_{28} \mathrm{H}_{20} \mathrm{~S}_{2} \mathrm{O}_{2}$, ist ein farbluser Körper. Das Reactionsproduct Dimethylcoerbithiën, $\mathrm{C}_{28} \mathrm{HI}_{18} \mathrm{~S}_{2}$, ist ein gelbrother Körper, der sich mit starker, grüner Fluorescenz löst und leicht wieder zu den Coerbithioniumsalzen oxydiren lässt. I)ie früher beschriebenen Erscheinungen wiederbolen sich genau in der gleichen Weise, indem man die gefärbten Verbindungen bald in die wasserlösliche Salzform, bald in die ätherlösliche Bithiënform überführen kann.

Was das Coerdiamidin ron Bayer $₫ \mathrm{Co}$. anbetrifft, so haben wir sämmtliche Angaben bei Wiederholung der Patentvorschrift bestätigt gefunden auch eine stimmende Analyse der Base erhalten. Die Salze sind blau, die Base violettroth. Das um zwei Wasserstoffatome reichere Reductionsproduct orange mit starker Fluorescenz und geht leicht an der Luft in das Coerbiamidin zurück; es ist weder in Alkalien noch in Säuren löslich. Die Bildung des Stickstoffisologen bei der Synthese rrfolgt in weit besseren Ausbeuten als die der Oxonium- und Thioniumisologen.

16. Zur Theorie der conjugirten Ringsysteme und der springenden Bindungen;

ron $H$. Decher.

In der Abbandlung über die Beziehungen des doppelt gebundenen Koblenstoffs zum Stickstoff, Sauerstoff und Schwefel I (Ber. d. deutsch. chem. Ges. 38, 2494) habe ich eine Anwendung 
und höhere homologe und isologe 1yryliumringe. 339

der Thiele'schen Anschauung auf die Addition von Säuren an zwei im Molekül weit auseinanderliegende Atome unter innerem Bindungswechsel versucht. Verhältnisse, die auf ähnliche Ursachen zurlickzuführen waren, traten bei den Uebergängen der Coeroxoniumsalze (I) in Coeroxenole (II) (und den gleichen Vorgängen in den isologen Reihen) unter dem Einflusse von Oxydations- und Reductionsmitteln auf.



Noch auffallender und durchsichtiger traten diese Erscheinungen aus dem Rahmen der gewöhnlich bei den bochmolekularen Körpern so träge verlaufenden Reactionen bei dem Uebergange der soeben geschilderten Coerbioxen, Coerbithiënen und dem Binaphtylenbioxyd $z u$ den entsprechenden Bioxonium- und Bithioniumsalzen (und umgekehrt) hervor. Ich habe die Erklärung dieser Erscheinungen in einer kurzen Notiz über conjugirte Dioniumsysteme ${ }^{32}$ ) publicirt, auf welche hier verwiesen sein soll, da sie die Ergänzung des mitgetheilten Versuchsmaterials bildet. Seit ihrem Erscheinen sind aber neue Thatsachen bekannt geworden, die ein höchst willkommenes Material zur Bestätigung der Theorie der conjugirten Ringsysteme bilden.

82) Ber. d. deutsch. chem. Ges. 39, 3069--71. 
Es ist dies erstens die Darstellung des 2,6-Naphtochinons durch Willstätter. Vergleicht man das Atomskelett des Binaphtylenbioxyds mit dem des 2, 6 -Naphtochinons und ihre Uebergänge zum Hydrochinon einerseits und zum Bioxoniumkörper andererseits, so sieht man, dass in der complexen Reihe sich das Skelett der einfachen jedesmal wiederholt und das Umspringen der entsprechenden Bindgungen nach dem gleichen Sehema erfolgt.

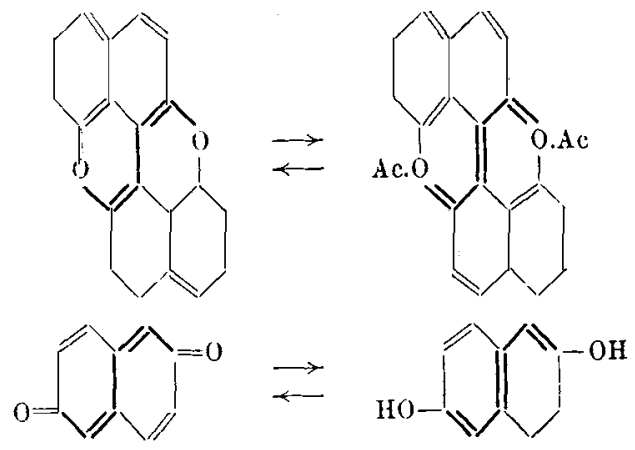

Ich hatte auch auf Grund der Betrachtungen der Umwandlungen des Dinaphthylenoxyds die Wahrscheinlichkeit der Existenz des Zweikernnaphtochinons (2: (3) abgeleitet und darin die Erklärung für die eigenthümlichen Beziehungen, die bei den Substitutionserscheinungen der 2-Derivate des Chinolins und des Naphtalins zu Tage treten und zwischen der 2,6-Stellung eine ähnliche Beziehung wie die Parastellung im Benzol erkennen lassen, gefunden. Durch die schönen Versuche von Willstätter ist die Conjugation der 2,6-Stellung in einfachster Weise zur Darstellung gebracht.

Ich hatte auch angedeutet, dass in den höheren complexen Ringen sich die Conjugation von weit auseinanderstehenden Gruppen durch ein System von Ringen hindurch auffinden lassen wird. Ich habe hier im Auge eine Anzabl von Küpenfarbstoffen, mebrere Anthracenringe zusammengeschwoisst enthaltend, die nach René Bohn's Vorgange sowobl von der Badischen Anilin- und Sodafabrik, als auch von den Farbenfabriken vorm. 
Bayer \& Co. dargestellt und pateutirt worden sind, ein Gebiet, das durch die umfangreichen Untersuchungen von Scholl theoretisch aufgeklärt und durch Syntbesen weiter ausgebaut worden ist. Mir ist sowohl die Aebnlichkeit der Atomskelette dieser Kupenfarbstoffe als auch die Analogie der Vorgänge der Küpenreactionen mit dem beim Coerbioxen und Coerbithiëp beobachteten, was ausserordentliche Leichtigkeit in Farbenwechsel anbetrifft, aufgefallen. Um ein concretes Beispiel zu nehmen, weise ich auf das neulich von Scholl eingehend untersuchte Pyranthren, dessen Constitution ron demselben auch einwandfrei durch eine gelungene Synthese festgelegt worden ist, hin. Wie

I.

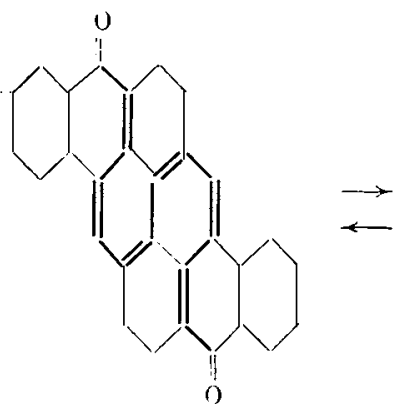

II.

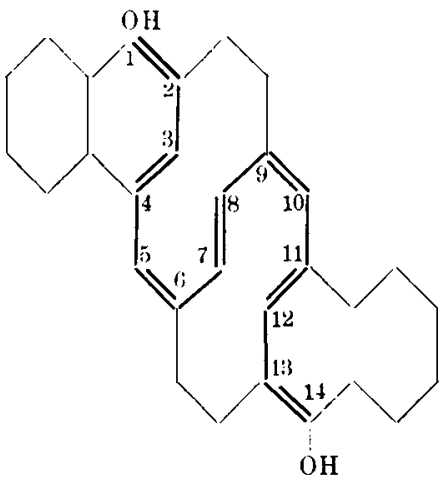

1. $\mathrm{O}=\mathrm{C}-\mathrm{C}=\mathrm{C}-\mathrm{C}=\mathrm{C}-\mathrm{C}=\mathrm{C}-\mathrm{C}=\mathrm{C}-\mathrm{C}=\mathrm{C}-\mathrm{C}=\mathrm{C}-\mathrm{C}=\mathrm{O}$.

II. $\mathrm{HO}-\mathrm{C}=\mathrm{C}-\mathrm{C}=\mathrm{C}-\mathrm{C}=\mathrm{C}-\mathrm{C}=\mathrm{C}-\mathrm{C}=\mathrm{C} \quad \mathrm{C}=\mathrm{C}-\mathrm{C}=\mathrm{C}-\mathrm{OH}$.

Das zweite Schema ist so zu verstehen, dass durch die Partialvalenz der letzten Kohlenstoffatome der Kette die zweite Valenz des Sauerstoffs angezogen wird und in Folge dessen haftet der Wasserstoff am Sauerstoff lose.

Wie man aus den Formeln sieht, sind die Erscheinungen in der Küpe das Spiel conjugirter Ringsysteme.

Ich bin auch der Ueberzeugung, dass das fortschreitende Studium der durch die Technik zugänglich gewordenen hoch- 
342 Decker und r. Fellenberg, Leber Phenopyrylium etc. molekularen, stickstofffreien Küpenfarbstoffe und der höheren complexen, aromatischen Reihen uberhaupt, diese Theorie weiter bestätigen wird, und dass weder durch das wachsende Molekulargewicht noch dureh die Anzahl der zwischen die reagirenden Gruppen tretender Ringe die Geschwindigkeit noch der glatte Verlauf der intramolekularen Reactionen Einbusse erleiden wird. 